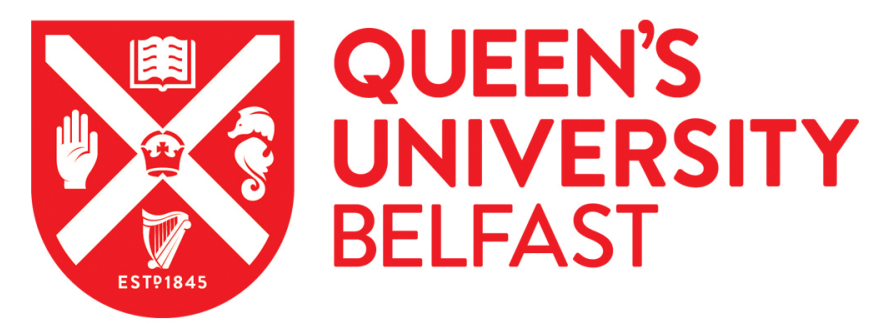

\title{
Low-energy excitations of a boson pair in a double-well trap
}

Murphy, D., \& McCann, J. (2008). Low-energy excitations of a boson pair in a double-well trap. Physical Review A, 77(6), [063413]. https://doi.org/10.1103/PhysRevA.77.063413

\author{
Published in: \\ Physical Review A
}

Document Version:

Publisher's PDF, also known as Version of record

Queen's University Belfast - Research Portal:

Link to publication record in Queen's University Belfast Research Portal

Publisher rights

(C) 2008 The American Physical Society

\section{General rights}

Copyright for the publications made accessible via the Queen's University Belfast Research Portal is retained by the author(s) and / or other copyright owners and it is a condition of accessing these publications that users recognise and abide by the legal requirements associated with these rights.

Take down policy

The Research Portal is Queen's institutional repository that provides access to Queen's research output. Every effort has been made to ensure that content in the Research Portal does not infringe any person's rights, or applicable UK laws. If you discover content in the Research Portal that you believe breaches copyright or violates any law, please contact openaccess@qub.ac.uk. 


\title{
Low-energy excitations of a boson pair in a double-well trap
}

\author{
D. S. Murphy and J. F. McCann* \\ Centre for Theoretical Atomic, Molecular and Optical Physics, The Queen's University Belfast, Belfast, BT7 1NN, United Kingdom
}

(Received 2 March 2008; published 12 June 2008)

\begin{abstract}
The states of a boson pair in a one-dimensional double-well potential are investigated. Properties of the ground and lowest excited states of this system are studied, including the two-particle wave function, momentum pair distribution, and entanglement. The effects of varying both the barrier height and the effective interaction strength are investigated.
\end{abstract}

DOI: $10.1103 /$ PhysRevA.77.063413

PACS number(s): 37.10.Gh, 05.30.Jp, 03.67.Mn, 37.10.Jk

\section{INTRODUCTION}

Ensembles of ultracold, trapped atoms provide an ideal test system for the study of fundamental quantum principles. The manipulation of atoms with photons [1], has given rise to the experimental realization of Bose-Einstein condensation (BEC) [2-4] and, more recently, the trapping and manipulation of condensates using optical lattice potentials [5-8]. The weak coupling of neutral atoms to their environment mean that this system of cold neutral atoms, confined by a periodic potential, may prove useful in the investigation of primitive quantum-information processing [9]. Indeed, such systems have already been used to carry out a two-qubit entangling operation $[10,11]$, thereby realizing the crucial controlled-NOT (CNOT) gate. At the same time, the spatially periodic nature of the system makes it ideal for the detailed study of solid-state Hamiltonians [12-14]. The benefit of this artificial system, in this regard, lies in the fact that the experimentalist can easily vary external control parameters (e.g., laser intensity or wavelength), thereby varying particular parameters of the system Hamiltonian. A degree of control that is not generally afforded to typical solid-state systems.

The dynamics of a system of ultracold atoms, confined by an optical lattice potential, can be accurately described within the framework of the Bose-Hubbard model $[12,13]$. In this model the system Hamiltonian is parametrized by the tunneling strength between adjacent lattice sites, $J$, and the on-site interaction energy, $U$. The Hamiltonian describing the system dynamics can then be written as

$$
\hat{H}=J \sum_{\langle i, j\rangle} \hat{b}_{i}^{\dagger} \hat{b}_{j}+\sum_{i} \epsilon_{i} \hat{n}_{i}+U \sum_{i} \hat{n}_{i}\left(\hat{n}_{i}-1\right),
$$

where $\hat{b}_{i}^{(\dagger)}$ is the annihilation (creation) operator for an atom at the lattice site $i$ and $\hat{n}_{i}=\hat{b}_{i}^{\dagger} \hat{b}_{i}$ is the number operator for that site. Parameter $\epsilon_{i}$ is the single-particle energy at lattice site $i$ and will vary with $i$ for an inhomogeneous lattice. Implicit in this model is the assumption that the dynamics of the system is dominated by single- and two-particle effects. In this way, the system of two, confined, interacting particles represents the fundamental building block for the understanding of these many-body systems. Furthermore, continual advance-

\footnotetext{
*j.f.mccann@qub.ac.uk
}

ment in optical lattice technology means that it has become possible to confine small numbers of atoms (e.g., 1 or 2) at individual lattice sites, effectively realizing a system of two trapped atoms.

For low-energy collisions the particle interactions can be accurately represented within the pseudopotential approximation [15]. The eigenstates for a system of two particles, interacting via a pseudopotential, can be determined analytically for both isotropic [16] and anisotropic [17] harmonic traps. Under such confinement, the free-space pseudopotential approximation is found to be sufficiently accurate provided the length scale associated with the particle-particle interactions $(a)$ is short compared to the length scale of the confining potential $(L)$ [18]. For the case in which $a$ and $L$ are comparable, one may introduce an energy-dependent scattering length and solve for the eigenenergies of the system self-consistently [19-21].

In addition to providing small numbers of particles at individual lattice sites, optical lattice experiments also allow for the realization of quasi-one-dimensional and quasi-twodimensional systems $[22,23]$. Simply increasing the confining potential steeply in one or two of the transverse directions will effectively freeze out the corresponding degrees of freedom $[24,25]$. Such systems of reduced dimensionality can also be achieved using optical or magnetic atom waveguides. The theoretical treatment of the particle-particle interactions in such low-dimensional geometries has been previously considered. For a quasi-one-dimensional (quasi1D) system it was found that the scattering could be treated in terms of a $1 \mathrm{D}$, zero-ranged $\delta$ potential, renormalized according to the confining potential [26]. The physical realization of such quasi-1D trap geometries and recent advances in the tuning of atomic interactions using Feshbach resonances have permitted the study of previously inaccessible regimes. Notably, the 1D system of impenetrable bosons, or so-called Tonks-Girardeau gas $[27,28]$, has commanded considerable experimental $[22,23]$ and theoretical [29-34] interest in recent years.

In [34] the detailed theoretical study of two interacting particles in a $\delta$-split harmonic potential was considered. The numerical techniques $[35,36]$, employed in [34] to study the $\delta$-split trap potential can be easily adapted to other types of confining potentials. In the current paper we utilize these same numerical techniques to study a prototypical two-well trap, defined by $V(x)=A\left(x^{4}-\kappa x^{2}\right)$. The eigenspectrum for this two-particle system is studied and properties of the ground and lowest excited states are investigated for varying 
of the barrier height (dictated by $\kappa$ ) and the strength of the particle-particle interactions. Particular consideration is given to the similarities observed between the ground-state structure in this prototypical two-well potential and that of the $\delta$-split potential [34].

Similar numerical studies of ultracold few-boson systems have been recently reported [37-39]. In this work the authors use a multiconfigurational time-dependent Hartree (MCTDH) method to study systems of several bosons in a double-well trap, with narrow width Gaussians used to represent both the central splitting potential and the interparticle potential. Where comparison is possible, the results of this numerical MCTDH study demonstrate qualitative similarity to the results of the present study.

The remainder of this paper is organized as follows. In Sec. II we outline the Hamiltonian that shall be considered, for two particles confined by a quasi-1D double-well potential. In Sec. III we provide a brief outline of the DVR techniques used to investigate this system. The energy level spectrum for the single- and two-particle systems is presented in Sec. IV, illustrating how the spectrum is influenced by barrier height and interaction strength. In Sec. V we examine various properties of the two-particle ground state. The properties considered include the ground-state wave function, momentum distributions (Sec. V A), and von Neumann entropy of the reduced single-particle density matrix (Sec. V B). Particular emphasis is given to how these properties may be influenced by varying the experimentally controllable parameters of barrier height and interaction strength. In Sec. VI we systematically examine these same properties for the lowest excited states of this system. Finally, in Sec. VII we summarize our findings and make some concluding remarks.

\section{SYSTEM HAMILTONIAN}

Consider a system of two interacting particles confined in two dimensions by means of a tight harmonic potential, having trapping frequency $\omega_{\perp}$ and associated length scale $d_{\perp}$ $=\sqrt{\hbar / m \omega_{\perp}}$. In the remaining third dimension, the confining potential is, relatively, loose and has the form

$$
V(x)=A\left(x^{4}-\kappa x^{2}\right) .
$$

The parameters $A$ and $\kappa$ determine the precise form of the double-well potential. It is straightforward to verify that the two minima of this double-well potential are located at $x_{\min }$ $= \pm \sqrt{\kappa / 2}$, with the potential at these minima being $V\left(x_{\min }\right)$ $=-A \kappa^{2} / 4$. The well separation, $x_{\min }$, and the barrier height, $V\left(x_{\min }\right)$, are controlled by the parameter $\kappa$.

As a result of the large energy level separation, associated with the transverse eigenstates $\left(\hbar \omega_{\perp}\right)$, the transverse motion of the particles is frozen out. In this way the particles are confined to the lowest motional state in each of these transverse directions. In this case the system is quasi-1D and may be effectively described by

$$
H=\sum_{i=1,2}\left(-\frac{\hbar^{2}}{2 m} \frac{\partial^{2}}{\partial x_{i}^{2}}+A\left(x_{i}^{4}-\kappa x_{i}^{2}\right)\right)+g_{1 \mathrm{D}} \delta\left(x_{2}-x_{1}\right) .
$$

Here, $m$ is the mass, and $x_{1}$ and $x_{2}$ are the coordinates of atoms 1 and 2 , respectively. The quantity $g_{1 \mathrm{D}}$ represents the particle-particle interaction strength, and is related to the 1D $s$-wave scattering length $\left(a_{1 \mathrm{D}}\right)$ through $g_{1 \mathrm{D}}=-2 \hbar^{2} / m a_{1 \mathrm{D}}$. In turn, $a_{1 \mathrm{D}}$ is related to the three-dimensional (3D) $s$-wave scattering length, $a_{3 \mathrm{D}}$, through $a_{1 \mathrm{D}}=-d_{\perp}^{2} / 2 a_{3 \mathrm{D}}(1$ $\left.-C a_{3 \mathrm{D}} / d_{\perp}\right)$, where $C$ is a constant and has approximate value $C=1.4603$ [26].

In the limit of tight confinement the free-space pseudopotential approximation, for the particle-particle interactions, becomes compromised $[18,19]$. In this case, one may obtain the eigenenergies for the system by employing an energydependent scattering length and solving for the energy eigenvalues self-consistently $[20,21,40]$. For current purposes it is supposed that we are in the regime for which the pseudopotential approximation is still valid and the 1D collisional coupling, $g_{1 \mathrm{D}}$, acts as a parameter for the system.

The aim is to study the eigenvalues and eigenvectors for the two-dimensional (2D) Hamiltonian given in Eq. (3). To facilitate this we introduce the scaling $x_{i}=\alpha \bar{x}_{i}$ for $i=1,2$. Under this rescaling the time-independent Schrödinger equation (TISE) can be written as

$$
\bar{H} \Psi_{i}\left(\bar{x}_{1}, \bar{x}_{2}\right)=\bar{E}_{i} \Psi_{i}\left(\bar{x}_{1}, \bar{x}_{2}\right),
$$

where

$$
\bar{H}=\frac{m \alpha^{2}}{\hbar^{2}} H=\sum_{i=1,2}\left(\frac{1}{2} \frac{\partial^{2}}{\partial \bar{x}_{i}^{2}}+\left(\bar{x}_{i}^{4}-\bar{\kappa} \bar{x}_{i}^{2}\right)\right)+\bar{g}_{1 \mathrm{D}} \delta\left(\bar{x}_{2}-\bar{x}_{1}\right) .
$$

Here the scaling factor, $\alpha$, has been chosen such that

$$
\frac{A m \alpha^{6}}{\hbar^{2}}=1
$$

Consequently,

$$
\begin{gathered}
\bar{\kappa}=\left(\frac{A m}{\hbar^{2}}\right)^{1 / 3} \kappa, \\
\bar{g}_{1 \mathrm{D}}=\frac{m}{\hbar^{2}}\left(\frac{\hbar^{2}}{A m}\right)^{1 / 6} g_{1 \mathrm{D}}, \\
\bar{E}=\frac{m}{\hbar^{2}}\left(\frac{\hbar^{2}}{A m}\right)^{1 / 3} E .
\end{gathered}
$$

For convenience we shall drop the bar on all quantities and use, exclusively, the scaled quantities just described.

\section{NUMERICAL TECHNIQUE}

In this paper we use a numerical discretization scheme to study the properties of the ground and lowest excited states of the two-boson system, as a function of the interaction strength $\left(g_{1 \mathrm{D}}\right)$ and the barrier height $(\kappa)$.

Discretization of the spatial coordinates $x_{1}$ and $x_{2}$ is achieved by means of a discrete variable representation (DVR) [35,36]. The two-particle wave function is represented by the direct product 


$$
\Psi\left(x_{1}, x_{2}\right)=\sum_{i, j=1}^{N} \Psi_{i j} f_{i}\left(x_{1}\right) f_{j}\left(x_{2}\right) .
$$

Here $\Psi_{i j}$ is the value of the two-particle wave function at the mesh point $\left(x_{1}=q_{i}, x_{2}=q_{j}\right)$, with $i, j=1,2, \ldots, N$. Clearly, these mesh points are finite in number and will be restricted to some region in $\left(x_{1}, x_{2}\right)$ space, defined by the boundaries $a$ and $b$, such that

$$
a<q_{i}<b, \quad i=1,2, \ldots, N .
$$

The values, $\Psi_{i j}$, play the role of variational parameters to be found and the $f_{i}(q)$ are a set of $N$ Lagrange functions which have the property that they are localized about the mesh points $q_{1}, q_{2}, \ldots, q_{N}$. In addition to satisfying the usual interpolation conditions,

$$
f_{i}\left(q_{j}\right)=\delta_{i j} \quad \forall i, j,
$$

one also requires that these Lagrange functions satisfy the orthogonality condition

$$
\int_{a}^{b} f_{i}^{*}(q) f_{j}(q) d q=\lambda_{i} \delta_{i j}
$$

Here $\lambda_{i}$ are the generalized Christoffel numbers associated with the mesh [35], and

$$
\lambda_{i}=1 \quad \forall i,
$$

for the Cartesian mesh considered in this work. For this Cartesian mesh the Lagrange functions are given by

$$
f_{i}(q)=\frac{1}{N} \frac{\sin [\pi(q-i)]}{\sin [\pi(q-i) / N]} .
$$

Using the basis expansion of Eq. (7) in the Schrödinger equation (4), results in a discrete eigenvalue problem that can be solved using standard linear algebra techniques.

For the results presented in this paper, a Cartesian DVR [35] is used to discretize the spatial coordinates $x_{1}$ and $x_{2}$. The discretization scheme used in most calculations employs $N=61$ mesh points in each dimension with a mesh spacing of $h=0.16$. For the wave-function plots a finer mesh is used to improve resolution within the figures $(N=81$ and $h=0.14)$. However, it is noted that any numerical results presented have been verified to be well converged with respect to variations in the mesh parameters $N$ and $h$.

\section{ENERGY SPECTRUM}

The eigenspectra for the single- and two-particle system are obtained, subject to the scaling introduced in Sec. II. Consideration is limited to the four lowest eigenvalues of the two-particle system (i.e., the lowest band), in particular we are interested in the behavior as the parameters $\kappa$ and $g_{1 \mathrm{D}}$ are varied.

\section{A. Single-particle spectrum}

Subject to the scaling introduced in Sec. II the TISE for the single-particle system is simply

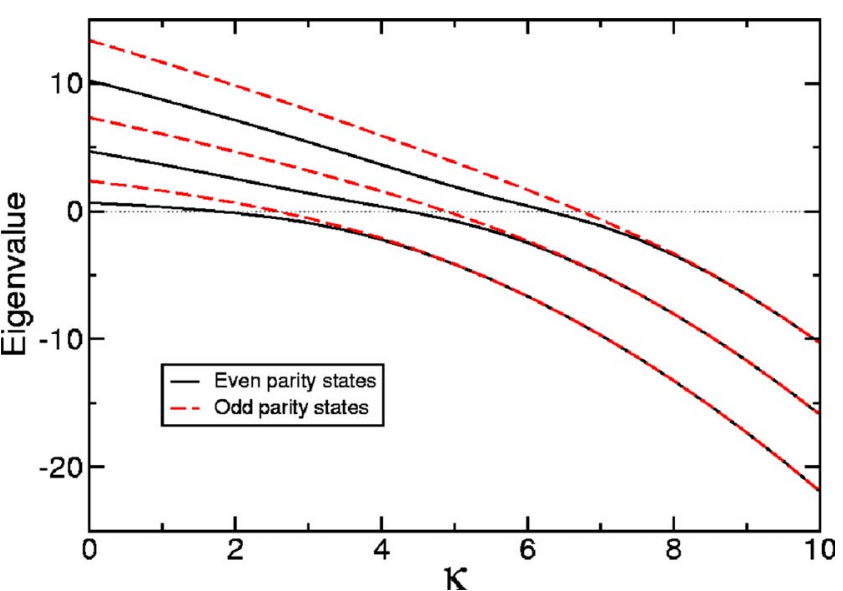

FIG. 1. (Color online) Lowest-energy eigenvalues for a single particle in the potential $V(x)=x^{4}-\kappa x^{2}$. The eigenstates alternate between states of odd parity (dashed lines) and even parity (solid lines). As the barrier is introduced these states pair up.

$$
\left(-\frac{1}{2} \frac{\partial^{2}}{\partial x^{2}}+\left(x^{4}-\kappa x^{2}\right)\right) u_{i}(\kappa ; x)=E_{i}^{\text {single }}(\kappa) u_{i}(\kappa ; x) .
$$

The single-particle spectrum is presented in Fig. 1. One can see that as the parameter $\kappa$ is increased the lowest eigenvalues are pulled downwards in energy as $V\left(x_{\min }\right)$ becomes increasingly negative. At the same time one observes the degeneracy of energy levels as $\kappa$ is increased.

\section{B. Two-particle spectrum}

Extending consideration to the two-particle spectrum, we focus attention on the two-particle eigenstates belonging to the lowest band. Denoting the $i$ th eigenstate of the twoparticle system by $\Psi_{i}$, the eigenstates for the lowest band are then denoted by $\Psi_{0}, \Psi_{1}, \Psi_{2}$, and $\Psi_{3}$ (see Sec. VI for details). This lowest band corresponds to the four lowest levels in Fig. 2(a), representing the two-particle system in the absence of interactions. In this noninteracting regime the two-particle eigenstates, under exchange symmetry, are

$$
\begin{gathered}
\Psi_{0}^{\mathrm{ni}}\left(\kappa ; x_{1}, x_{2}\right)=u_{0}\left(\kappa ; x_{1}\right) u_{0}\left(\kappa ; x_{2}\right), \\
\Psi_{2,1}^{\mathrm{ni}}\left(\kappa ; x_{1}, x_{2}\right)=\frac{1}{\sqrt{2}}\left[u_{0}\left(\kappa ; x_{1}\right) u_{1}\left(\kappa ; x_{2}\right) \pm u_{1}\left(\kappa ; x_{1}\right) u_{0}\left(\kappa ; x_{2}\right)\right], \\
\Psi_{3}^{\mathrm{ni}}\left(\kappa ; x_{1}, x_{2}\right)=u_{1}\left(\kappa ; x_{1}\right) u_{1}\left(\kappa ; x_{2}\right),
\end{gathered}
$$

with the two-particle eigenenergies given by corresponding combinations of the single-particle energies, $E_{i}^{\text {single }}(\kappa)$. From Fig. 2(a), one notes that as $\kappa$ is increased all two-particle eigenstates in the lowest band become degenerate. This degeneracy follows automatically from the degeneracy of the states $u_{0}(\kappa ; x)$ and $u_{1}(\kappa ; x)$ seen in the single-particle case (see Fig. 1). The states, which are symmetric (solid lines) and antisymmetric (dashed lines) under exchange, are indicated, corresponding to boson and fermion pairs.

The effect of introducing interactions between the two bosons is displayed in Figs. 2(b)-2(d). In Fig. 2(b) a scaled 


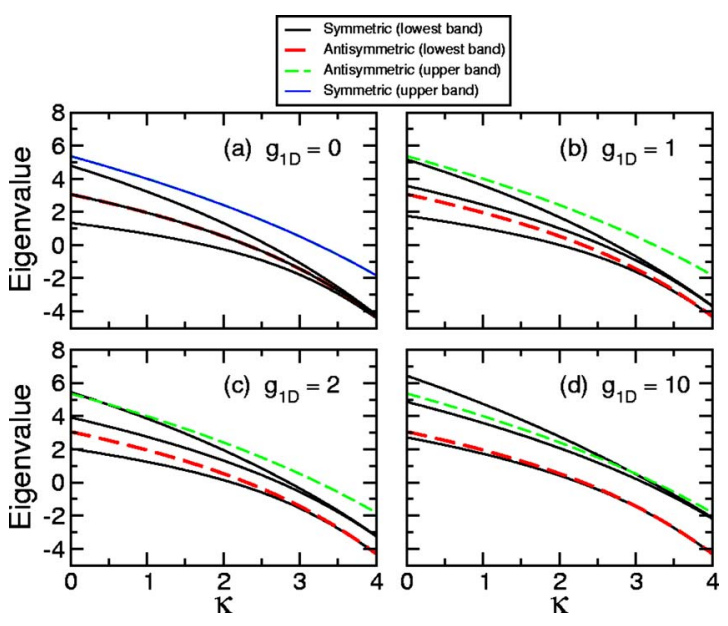

FIG. 2. (Color online) Lowest-energy eigenvalues for the system of particles in a double-well potential of the form $V(x)=x^{4}$ $-\kappa x^{2}$, as a function of the barrier height, $\kappa$. The spectrum is displayed for four different values of interaction strength: $g_{1 \mathrm{D}}=0$ (a), $g_{1 \mathrm{D}}=1(\mathrm{~b}), g_{1 \mathrm{D}}=2(\mathrm{c})$, and $g_{1 \mathrm{D}}=10(\mathrm{~d})$. Introduction of the interaction coupling has the effect of shifting the symmetric states (solid lines) upwards in energy, while the antisymmetric states (dashed lines) remain unaffected.

interaction coupling of $g_{1 \mathrm{D}}=1$ is considered. The symmetric states are shifted upwards in energy as a result of the repulsive interactions while the antisymmetric states remain unaltered. In the limit of large $\kappa$ one now observes two pairs of degenerate levels, as opposed to the set of four degenerate states seen in the noninteracting case. The energy separation of these two pairs of levels is monotonically increasing with increasing $\kappa$. Increasing the interaction coupling further, Fig. 2 (c), leads to one of the symmetric states being promoted above the higher-lying antisymmetric state for small $\kappa$, but with increased $\kappa$ the normal ordering is restored. Finally, Fig. 2(d) depicts the same spectrum in the limit of strong repulsion: $g_{1 \mathrm{D}}=10$. The lowest symmetric state now follows closely the energy profile of the lowest antisymmetric state. This feature is a universal property for a system of strongly interacting bosonic particles in $1 \mathrm{D}$. In the limit of $g_{1 \mathrm{D}} \rightarrow \infty$ the bosonic particles become impenetrable, and one enters the so-called Tonks-Girardeau regime [27,28]. The FermiBose mapping $[28,29]$ allows, for example, the ground state of the bosonic system to be given by

$$
\Psi_{0}\left(x_{1}, x_{2}\right)=\left|\Psi_{1}^{\mathrm{ni}}\left(x_{1}, x_{2}\right)\right| .
$$

The similarity of the energy of the symmetric ground state and the lowest antisymmetric state, seen in Fig. 2(d), is an indication that the system is approaching this TonksGirardeau regime.

\section{GROUND-STATE PROPERTIES}

The ground-state wave function, $\Psi_{0}\left(x_{1}, x_{2}\right)$, for two interacting particles, is presented in Fig. 3. The individual color scale plots will be referenced using standard (row, column) matrix notation.

In the noninteracting case and for $\kappa=0$, plot $(1,1)$, the wave function is distributed fairly isotropically about the

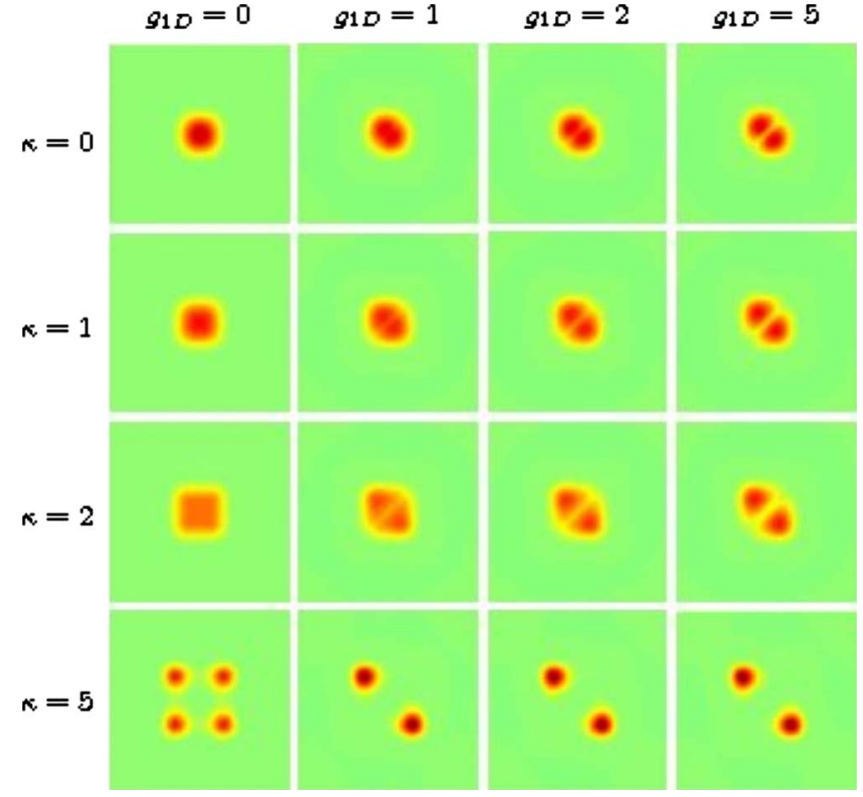

FIG. 3. (Color online) Ground-state wave function, $\Psi_{0}\left(x_{1}, x_{2}\right)$, for a boson pair in a double-well potential. The different columns represent different values for the interaction coupling. The values considered are $g_{1 \mathrm{D}}=0$ (column 1), $g_{1 \mathrm{D}}=1$ (column 2), $g_{1 \mathrm{D}}=2(\mathrm{col}-$ umn 3 ), and $g_{1 \mathrm{D}}=5$ (column 4 ). In each case the effect of varying the barrier height is illustrated down a given column. Row 1 corresponds to $\kappa=0$, row 2 to $\kappa=1$, row 3 to $\kappa=2$, and row 4 to $\kappa=5$. Each individual plot spans the range $-5.6<x_{1}, x_{2}<5.6$, where $x_{1}$ is along the horizontal axis and $x_{2}$ is along the vertical. The quantities $x_{1}, x_{2}, \kappa$, and $g_{1 \mathrm{D}}$ are given in the scaled units introduced in Sec. II.

center of the trap. Moving down this column, increasing $\kappa$, the wave function expands slightly in both dimensions and takes on a more rectilinear appearance, e.g., plot $(3,1)$. For small values of $\kappa$, the potential resembles that of a square well. The $x^{4}$ term gives rise to a steep boundary and the distribution of the two (independent) particles will be quite uniform, leading to the distribution seen in $(3,1)$. As the value of $\kappa$ is increased the wave function begins to segregate into four quadrants with suppression in the region of the barrier (i.e., along the lines $x_{1}=0$ and $x_{2}=0$ ). This effect is seen, quite markedly, in plot $(4,1)$. Considering the energy level spectrum in Fig. 2(a) one can see that for $\kappa=2$ one has not yet reached the insulator limit, whereas for $\kappa=5$ one is deep within this insulator regime, for which degeneracy is observed for the four lowest two-particle levels.

Turning to the fourth column of Fig. 3, plot $(1,4)$ shows the case of no barrier $(\kappa=0)$. The repulsive interaction precludes any overlap of the particles. The effect of increasing the barrier height to $\kappa=1, \kappa=2$, and $\kappa=5$ can be seen in plots $(4,2),(4,3)$, and $(4,4)$, respectively. Again, for small values of $\kappa$, the wave-function distribution expands slightly in $\left(x_{1}, x_{2}\right)$ space, but now the presence of repulsive interactions distorts the wave function along the line $x_{1}=-x_{2}$. In the insulator limit, as we have for $(4,4)$, one sees that the wave function has split into two clear lobes.

The behavior observed in Fig. 3 correlates closely to the behavior reported for the $\delta$-split potential in [34]: The segregation of the wave-function distribution into four quad- 
rants, and the vacancy of two of these quadrants owing to the introduction of repulsive interactions. These features are essentially generic for double-well systems.

\section{A. Momentum distribution}

The reduced single-particle density matrix (RSPDM) has proven to be an extremely useful mathematical construct in the analysis of pair correlations [41]. For the two-particle system considered here, the RSPDM, $\rho_{i}\left(x, x^{\prime}\right)$, for a given eigenstate, $\Psi_{i}\left(x_{1}, x_{2}\right)$, is defined to be

$$
\rho_{i}\left(x, x^{\prime}\right)=\int_{-\infty}^{+\infty} \Psi_{i}\left(x, x_{2}\right) \Psi_{i}\left(x^{\prime}, x_{2}\right) d x_{2} .
$$

This object has been analyzed in detail for the ground state of two particles in a $\delta$-split potential [34]. The behavior of $\rho_{0}\left(x, x^{\prime}\right)$ for the double-well, presented here, exhibits the same gross features as have been observed in [34] for the $\delta$-split trap problem. Instead, in this section we focus on the momentum distributions for this system.

The reciprocal momentum distribution for the $i$ th eigenstate, $n_{i}(k)$, is calculated from the corresponding reduced single-particle density, $\rho_{i}\left(x, x^{\prime}\right)$, through Fourier transform

$$
n_{i}(k) \equiv(2 \pi)^{-1} \int_{-\infty}^{+\infty} \int_{-\infty}^{+\infty} \rho_{i}\left(x, x^{\prime}\right) e^{-l k\left(x-x^{\prime}\right)} d x d x^{\prime},
$$

where $\int_{-\infty}^{+\infty} n_{i}(k) d k=1$. Equivalently, one may obtain the momentum distribution for this eigenstate by considering the diagonalization of $\rho_{i}\left(x, x^{\prime}\right)$. Specifically, the eigenvalue equation is

$$
\int_{-\infty}^{+\infty} \rho_{i}\left(x, x^{\prime}\right) \phi_{i j}\left(x^{\prime}\right) d x^{\prime}=\lambda_{i j} \phi_{i j}(x),
$$

where $\lambda_{i j}$, represents the fractional population of the natural orbital $\phi_{i j}(x)$ such that $\Sigma_{j} \lambda_{i j}=1$, for each $i$. Using numerical quadrature allows one to rewrite (18) as a linear equation. The momentum distribution, $n_{i}(k)$, may then be obtained from the relation

$$
n_{i}(k)=\sum_{j} \lambda_{i j}\left|\mu_{i j}(k)\right|^{2},
$$

where $\mu_{i j}(k)$ denotes the Fourier transform of the natural orbital $\phi_{i j}(x)$,

$$
\mu_{i j}(k)=\frac{1}{\sqrt{2 \pi}} \int_{-\infty}^{+\infty} \phi_{i j}(x) e^{-l k x} d x .
$$

The momentum distribution for the ground state is presented in Fig. 4. The distributions presented correspond to $g_{1 \mathrm{D}}=0$ (a), $g_{1 \mathrm{D}}=1$ (b), $g_{1 \mathrm{D}}=2$ (c), and $g_{1 \mathrm{D}}=5$ (d). Also within each figure, the distributions arising for several different values for the barrier height $(\kappa)$ are illustrated. In the noninteracting case, Fig. 4(a), one observes an initial peaked distribution for $\kappa=0$. Increasing $\kappa$ enhances the peak and narrows the distribution. With increasing barrier, the ground-state wave function adapts to spread over the available interval, leading to this reciprocal narrowing in momentum space. Further in-

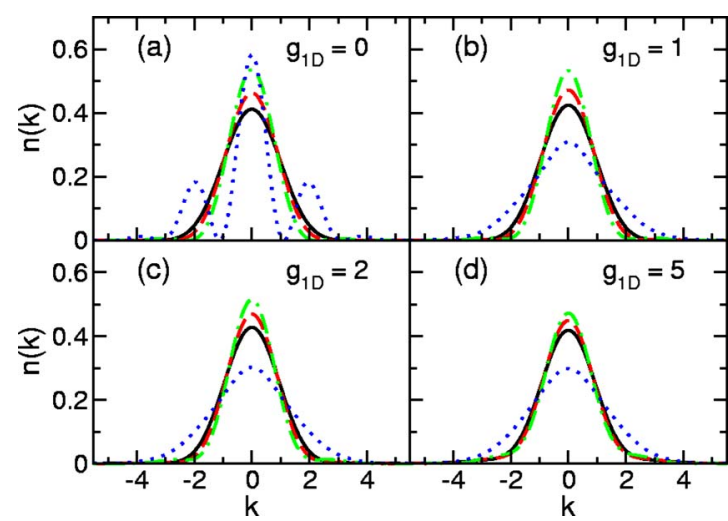

FIG. 4. (Color online) Momentum distribution for the ground state of a system of two bosons confined by a double-well potential. Four different values of interaction strength are considered: (a) $g_{1 \mathrm{D}}=0$, (b) $g_{1 \mathrm{D}}=1$, (c) $g_{1 \mathrm{D}}=2$, and $g_{1 \mathrm{D}}=5$. In each figure the effect of varying the barrier height is also illustrated. Barrier heights considered are $\kappa=0$ (solid line), $\kappa=1$ (dashed line), $\kappa=2$ (dotteddashed line), and $\kappa=5$ (dotted line).

crease in $\kappa$ means that the particles begin to experience the effect of the double-well. As the particles are noninteracting, the system displays a single-particle behavior. For a value of $\kappa=5$ (insulator regime) the particle splits between the wells and the momentum distribution displays prominent secondorder peaks, seen in Fig. 4(a). The momentum distributions can be observed by scattering or free expansion of the particles in the absence of a confining potential. From this perspective the second-order peaks correspond to the interference fringes that arise from two coherent matter wave sources.

Introducing an interaction encourages localization and has the effect of removing these secondary peaks. Once again, for small values of $\kappa$, e.g., $\kappa=1$ (dashed line) and $\kappa=2$ (dotted-dashed line), the momentum distribution becomes increasingly peaked and narrower. In the presence of interactions the particles are restricted to separate wells and the interference effects are lost. In addition, the localization of the particles leads to a broadening of the momentum distribution, as observed in Figs. 4(b)-4(d), for $\kappa=5$ (dotted line). It is also observed that, in the absence of any barrier, $\kappa=0$ (solid line), one sees the emergence of higher-energy wings for increasing interaction, $g_{1 \mathrm{D}}$. Similar high-energy wings have been reported in the TG regime for free space [26], and harmonic confinement [31].

\section{B. von Neumann entropy}

Entanglement is a fundamental expression of information content and is responsible for the increased efficiency of some quantum algorithms over their classical counterparts. Previous authors have shown that the von Neumann entropy of the RSPDM is a good measure of entanglement for a system of two bosons [42-44]. For the case of two indistinguishable particles, determination of whether or not the two subsystems are entangled requires that one considers both the von Neumann entropy of the reduced single-particle density matrix, and the Schmidt number, i.e., number of nonzero 


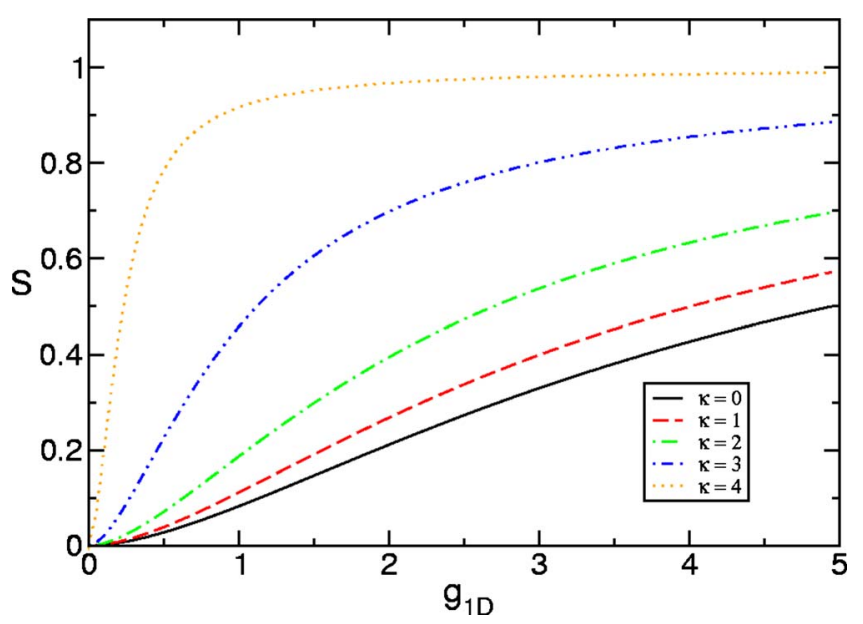

FIG. 5. (Color online) von Neumann entropy, $S$, as a function of the interaction strength, $g_{1 \mathrm{D}}$. This dependence is also illustrated for a number of different barrier heights: $\kappa=0$ (solid line), $\kappa=1$ (dashed line), $\kappa=2$ (dotted-dashed line), $\kappa=3$ (dotted-dotted-dashed line), and $\kappa=4$ (dotted line). One observes an increased sensitivity to $g_{1 \mathrm{D}}$ as the barrier is raised.

eigenvalues $\left(\lambda_{i j}\right)$ obtained in the diagonalization of $\rho_{i}$, Eq. (18) [45-47]. We use the von Neumann entropy to quantify the entanglement in the position coordinates, $x_{1}$ and $x_{2}$, of the particle pair.

Following the diagonalization of the reduced singleparticle density, Eq. (18), the von Neumann entropy for the $i$ th eigenstate of the two-particle system $\left(S_{i}\right)$ is obtained from

$$
S_{i}=-\sum_{j} \lambda_{i j} \log _{2} \lambda_{i j}
$$

\section{Variation of von Neumann entropy with interaction strength}

Variation of the von Neumann entropy with $g_{1 \mathrm{D}}$, for the ground state of this system, is plotted in Fig. 5. Examining the lowest solid line $(\kappa=0)$, when no interactions are present $\left(g_{1 \mathrm{D}}=0\right)$ then $S=0$ as one expects. The product states (with correct symmetrization) given in Eq. (14) represent the eigenstates of the noninteracting system. Introducing a small interaction has the effect of introducing correlations and results in a nonzero entropy. Increasing the interaction strength leads to an increasing entropy, saturating at $S \approx 1$, as for the harmonic potential $[34,44]$. This behavior can be related to fermionization. As the repulsive interactions increase, the system enters the TG regime. In this regime the ground state of the system can be represented by the corresponding system of two noninteracting fermions, with correct symmetrization. In terms of the eigenfunctions prescribed in Eq. (14), the ground state of the system is given by $\left|\Psi_{1}^{\mathrm{ni}}\left(\kappa ; x_{1}, x_{2}\right)\right|$ $=\left|\frac{1}{\sqrt{2}}\left[u_{0}\left(\kappa ; x_{1}\right) u_{1}\left(\kappa ; x_{2}\right)-u_{1}\left(\kappa ; x_{1}\right) u_{0}\left(\kappa ; x_{2}\right)\right]\right|$. The antisymmetric state, $\Psi_{1}$, in the presence of pointlike interactions, will always give $S=1$. The ground state of the system becomes degenerate with this antisymmetric state in the limit of hard-core interactions.

Figure 5 also displays the effect of increasing the barrier height, $\kappa$. As the system tends toward the insulator regime,
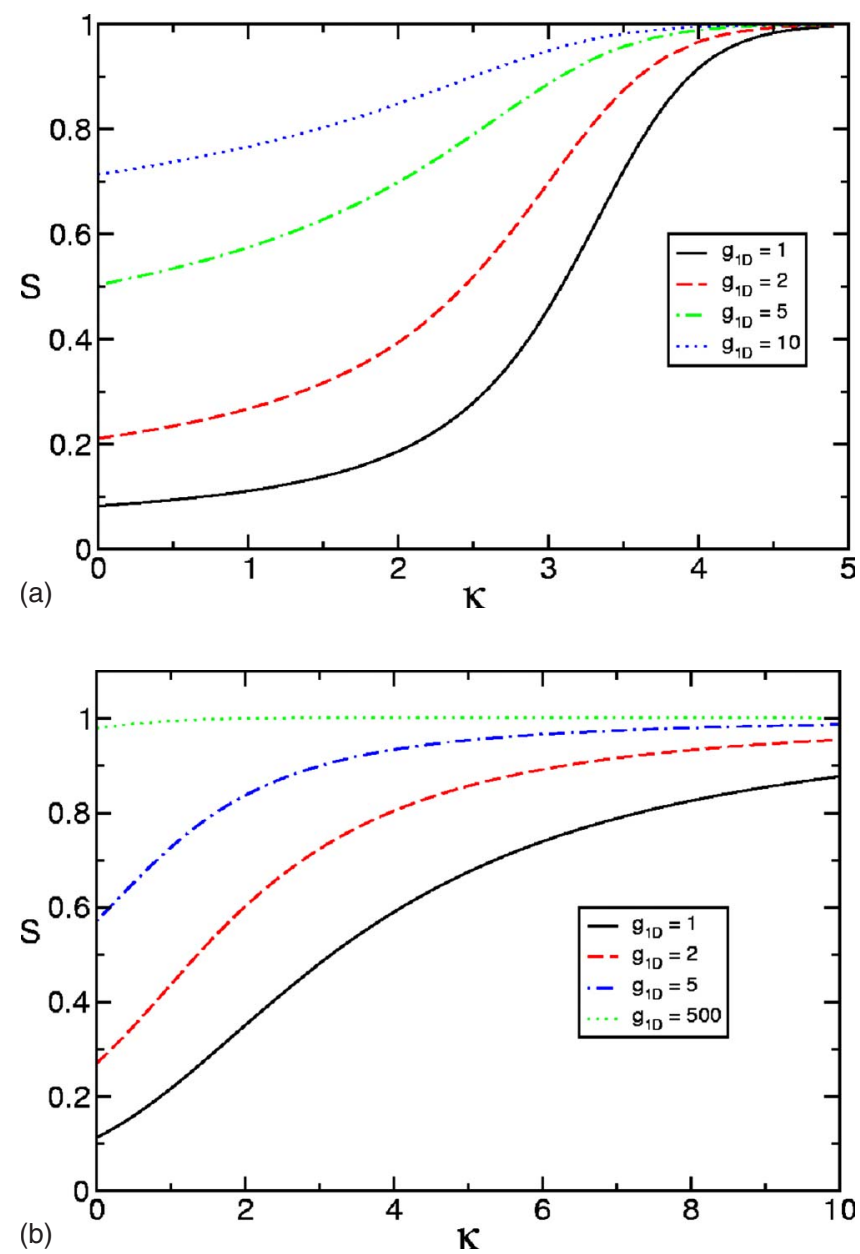

FIG. 6. (Color online) (a) von Neumann entropy, $S$, as a function of the barrier height, $\kappa$, for the double-well potential. The dependence is examined for a number of different interaction coupling strengths: $g_{1 \mathrm{D}}=1$ (solid line), $g_{1 \mathrm{D}}=2$ (dashed line), $g_{1 \mathrm{D}}=5$ (dotteddashed line), and $g_{1 \mathrm{D}}=10$ (dotted line). In all cases $S$ saturates at a value of unity in the limit $\kappa \rightarrow \infty$. (b) von Neumann entropy for the ground state of the $\delta$-split potential, as presented in [34]. For the present potential there is a more sudden transition to the Mottinsulator regime with increasing $\kappa$.

the entropy of the system becomes increasingly sensitive to changes in $g_{1 \mathrm{D}}$, about $g_{1 \mathrm{D}}=0$. This effect was also reported in [34] for the $\delta$-split trap, suggesting that this is another generic feature associated with double-well potentials. The increased barrier height reduces tunneling between the wells. For any increase in the interaction strength, the two-particle wave function will attempt to redistribute so as to minimize this interaction. However, with the increased barrier height the wave function is forced to remain more localized, and is restricted in its redistribution.

\section{Variation of von Neumann entropy with barrier height}

Variation of the von Neumann entropy with $\kappa$, for the ground state of this system, is plotted in Fig. 6(a). For ease of comparison, similar results for the $\delta$-split potential $\left[\frac{1}{2} x^{2}\right.$ $+\kappa \delta(x)$ ], studied in [34], are presented in Fig. 6(b). The basic trends for the double-well have a striking resemblance to 
those seen for the $\delta$-split trap [34]. Specifically, one observes that the initial entropy of the system (i.e., for $\kappa=0$ ) is dictated by the interaction strength of the system, $g_{1 \mathrm{D}}$. The larger is $g_{1 \mathrm{D}}$, the larger is the initial value of $S$, as is consistent with Fig. 5. Increasing the height of the barrier then has the effect of increasing the entropy of the system toward $S$ $=1$. In the limit of large barrier heights the entropy of the system saturates at $S=1$, regardless of the value of interaction strength (the notable exception being the noninteracting case, for which $S$ is identically equal to zero for all $\kappa$ ). This saturation at $S=1$ is interpreted as a loss of entanglement.

When the system enters the insulator limit there is an implicit exchange uncertainty in the state of the system, arising from the indistinguishable nature of the particles. There remains ongoing debate as to whether it is possible to use statistical correlations to perform any useful quantuminformation tasks [45-48]. In the present paper the interpretation adopted is that, in this insulator limit the state must be regarded as nonentangled. This diagnosis follows from the criteria set out in [45-47]. In the limit $\kappa \rightarrow \infty$ then $S \rightarrow 1$ and the Schmidt number can be seen to approach a value of 2 (not shown here). By the criteria outlined in [46], any state for which $S=1$ and with a Schmidt number of 2 must be regarded as nonentangled.

In contrast to the $\delta$-split trap, Fig. 6(b), the entropy dependence $S(\kappa)$ for the double-well system is quite sigmoidal. This is a feature of the double-well potential considered here. From Fig. 2 it can be seen that the system does not exhibit clear double-well behavior until $\kappa \geq 3$. For smaller values of $\kappa$ (i.e., $\kappa \lesssim 2$ ), variation of the barrier height only has a modest effect on the lower-energy states. However, for $\kappa \gtrsim 3$, the combined effect of an increasing barrier height and an increasing separation of the wells, leads to a rapid reduction in tunneling strength. In this way, these lowest levels are rapidly plunged into the insulator regime. Figure 2 suggests that the lowest-energy levels of the double-well potential rapidly transition into the insulator regime across the interval $2<\kappa$ $<3$. Correspondingly, from Fig. 6(a), one identifies $2<\kappa$ $<3$ as the interval over which the entropy makes its most rapid variation.

\section{3. von Neumann entropy in the Bose-Hubbard model}

One may examine the von Neumann entropy of the ground state within the formalism of the Bose-Hubbard model presented in Eq. (1). Using a Fock basis for the twoparticle system of the form $\left|n_{L} n_{R}\right\rangle$, where $n_{L(R)}$ represents the number of particles in the left (right) well, leads to three basis states: $|20\rangle,|11\rangle$, and $|02\rangle$.

Thus, in terms of this basis the Hamiltonian (1) may be written in matrix form as

$$
\hat{H}=\left(\begin{array}{ccc}
2 \epsilon+2 U & \sqrt{2} J & 0 \\
\sqrt{2} J & 2 \epsilon & \sqrt{2} J \\
0 & \sqrt{2} J & 2 \epsilon+2 U
\end{array}\right)
$$

with eigenvalues of this Hamiltonian that follow from some simple algebra

$$
\begin{gathered}
E_{-}=2 \epsilon+U-\sqrt{U^{2}+4 J^{2}}, \\
E_{\text {mid }}=2 \epsilon+2 U, \\
E_{+}=2 \epsilon+U+\sqrt{U^{2}+4 J^{2}} .
\end{gathered}
$$

The eigenvectors corresponding to the eigenvalues presented in Eq. (23) are found to be

$$
\left|\Psi_{\text {mid }}\right\rangle=\frac{1}{\sqrt{2}}\left(\begin{array}{c}
1 \\
0 \\
-1
\end{array}\right), \quad\left|\Psi_{ \pm}\right\rangle=\mathcal{N}_{ \pm}\left(\begin{array}{c}
1 \\
\frac{2 \sqrt{2} J}{U \pm \sqrt{U^{2}+4 J^{2}}} \\
1
\end{array}\right),
$$

where $\mathcal{N}_{ \pm}$represent normalization factors and $\left|\Psi_{\text {mid }}\right\rangle$ has odd inversion symmetry.

Given the two-particle ground state in the Fock basis, $\left|\Psi_{-}\right\rangle$, one may determine the reduced single-particle density matrix by tracing over the degrees of freedom of either particle. The single-particle basis states may be represented in the form $\left|n_{L} n_{R}\right\rangle$ as $|10\rangle$ and $|01\rangle$. In turn, the twoparticle basis states can be written in the symmetric form

$$
\begin{gathered}
|20\rangle=|10\rangle_{1} \otimes|10\rangle_{2}, \\
|11\rangle=\frac{1}{\sqrt{2}}\left[|10\rangle_{1} \otimes|01\rangle_{2}+|01\rangle_{1} \otimes|10\rangle_{2}\right], \\
|02\rangle=|01\rangle_{1} \otimes|01\rangle_{2} .
\end{gathered}
$$

The eigenvalues of this RSPDM are found to be

$$
\begin{aligned}
& \lambda_{1}=\mathcal{N}_{-}^{2}\left(1+\frac{4 J^{2}}{\left(U-\sqrt{U^{2}+4 J^{2}}\right)^{2}}-\frac{4 J}{U-\sqrt{U^{2}+4 J^{2}}}\right), \\
& \lambda_{2}=\mathcal{N}_{-}^{2}\left(1+\frac{4 J^{2}}{\left(U-\sqrt{U^{2}+4 J^{2}}\right)^{2}}+\frac{4 J}{U-\sqrt{U^{2}+4 J^{2}}}\right) .
\end{aligned}
$$

The variation of the ground-state entropy $(S)$ with the model parameters $J$ and $U$ is depicted as a surface plot in Fig. 7. It is noted that the qualitative behavior of the entropy displayed in Figs. 5 and 6 for varying $g_{1 \mathrm{D}}$ and $\kappa$, respectively, is reflected in the Bose-Hubbard model with variation of the parameters $U$ and $J$. This tight-binding approximation is poor in the limit $U / J \rightarrow 0$, but is an accurate representation in the insulator limit.

\section{EXCITED STATES}

Attention is now turned to the three lowest excited states which, together with the ground state, represent the lowestenergy band of the two-particle double-well system. In the noninteracting case, with spectrum depicted in Fig. 2(a), the three lowest excited states may be represented as given in Eq. (14), with one of these states being antisymmetric and two of them symmetric. Variation of parameters $\kappa$ and $g_{1 \mathrm{D}}$ 


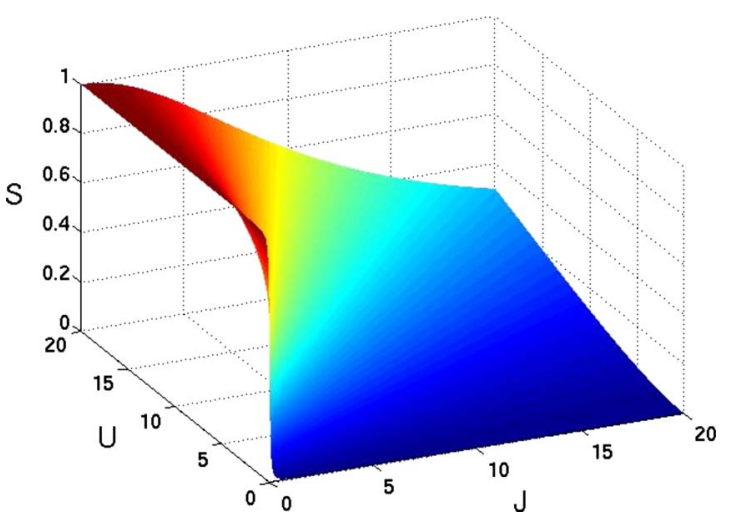

FIG. 7. (Color online) von Neumann entropy of the ground state as a function of the Hamiltonian parameters $J$ and $U$. The limit $J / U \rightarrow 0$ corresponds to the insulator limit and $U / J \rightarrow 0$ to the conductor limit.

can lead to reordering of the energy eigenvalues, as observed in Fig. 2. However, in this section, the study of the excited states of this two-particle system will be restricted to these three states of the lowest band, identifiable through their symmetry. Henceforth, the term first-excited state refers to the lowest-energy antisymmetric state $\left(\Psi_{1}\right)$, second-excited state refers to the second-lowest-energy symmetric state $\left(\Psi_{2}\right)$, and third-excited state refers to the third-lowest-lying symmetric state $\left(\Psi_{3}\right)$.

\section{A. Two-particle excitations}

The wave functions for $\Psi_{1,2,3}$ are represented, by means of color scale plots, in Figs. 8-10, respectively. Again, the standard (row, column) notation is used to reference individual subplots. The color scale is consistent across all wavefunction plots, permitting direct comparison between Figs. 3 and $8-10$.

Figure 8 represents the ground state for a system of two spin-aligned fermions $\left(\Psi_{1}\right)$, which is identically zero along the line $x_{1}=x_{2}$ and, thereby, unaffected by the zero-ranged interaction. Considering Fig. 8, moving along a given row (i.e., increasing repulsion for a fixed barrier), the wavefunction plots remain unchanged, illustrating the independence of this state with respect to interaction strength, $g_{1 \mathrm{D}}$. As $\kappa$ increases (i.e., down any column) the positive and negative lobes along the $x_{1}=-x_{2}$ diagonal become more widely separated indicating isolation into separate wells. Once again the wave-function density in these two quadrants correspond to the situation where particle 1 is in the left well $\left(x_{1}<0\right)$ and particle 2 is in the right $\left(x_{2}>0\right)$, and vice versa. In the limit of a large barrier, the ground state becomes degenerate with this antisymmetric state. The wave-function plots are almost identical (except for sign) for $\kappa=5$ as seen, for example, by comparing Fig. $3(4,4)$ and Fig. $8(4,4)$. Furthermore, as already discussed, one expects the ground state to become degenerate with this antisymmetric state in the limit of $g_{1 \mathrm{D}} \rightarrow \infty$, for all $\kappa$. This degeneracy is evidenced by comparing the fourth column in Fig. 3 to any column in Fig. 8. Even at this finite interaction strength $\left(g_{1 \mathrm{D}}=5\right)$ the equivalence of these two states is apparent. Finally, from

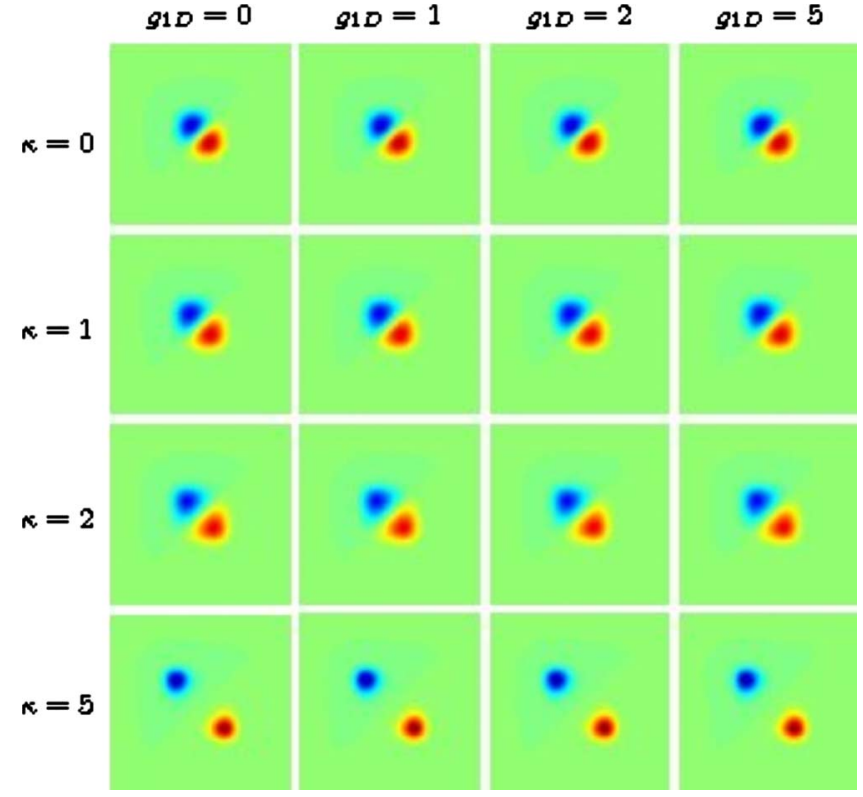

FIG. 8. (Color online) First-excited eigenfunction, $\Psi_{1}\left(x_{1}, x_{2}\right)$, for a pair of particles in a double-well potential. The color scale runs from blue (largest negative value) to red (largest positive value). The different columns represent different values for the interaction coupling. The values considered are $g_{1 \mathrm{D}}=0$ (column 1 ), $g_{1 \mathrm{D}}=1$ (column 2), $g_{1 \mathrm{D}}=2$ (column 3), and $g_{1 \mathrm{D}}=5$ (column 4). In each case the effect of varying the barrier height is illustrated down a given column. Row 1 corresponds to $\kappa=0$, row 2 to $\kappa=1$, row 3 to $\kappa=2$, and row 4 to $\kappa=5$. Notice how the antisymmetric state is completely independent of the interaction parameter, $g_{1 \mathrm{D}}$. Each individual plot spans the range $-5.6<x_{1}, x_{2}<5.6$, where the quantities $x_{1}, x_{2}, \kappa$, and $g_{1 \mathrm{D}}$ are given in the scaled units introduced in Sec. II.

each of the plots in Fig. 8 it is clear that this eigenstate is of odd parity, such that $\Psi_{1}\left(x_{1}, x_{2}\right)=-\Psi\left(-x_{1},-x_{2}\right)$.

Figure 9 depicts the second-excited state for the system of two bosons in a double-well potential and, as with $\Psi_{1}$, this state exhibits odd parity, $\Psi_{2}\left(x_{1}, x_{2}\right)=-\Psi_{2}\left(-x_{1},-x_{2}\right)$. The case of no barrier $(\kappa=0)$ and no interaction $\left(g_{1 \mathrm{D}}=0\right)$ is illustrated in Fig. $9(1,1)$. The eigenstate is composed of two lobes which correspond to both particles coexisting on the same side of the well. In the case of no interactions $\left(g_{1 \mathrm{D}}=0\right)$, illustrated in column 1, this symmetric eigenstate is degenerate with the antisymmetric state considered in Fig. 8. Repulsive interactions will tend to exclude the wave function from the line $x_{1}=x_{2}$ [e.g., compare $(1,1)$ to $(1,3)$ or $\left.(1,4)\right]$. In the Tonks limit this splits each of the upper-right and lower-left lobes. Considering the effect of the barrier in column 3 $\left(g_{1 \mathrm{D}}=2\right)$, the initial wave function demonstrates the doublelobe structure. As the barrier is increased to $\kappa=1,(2,3)$, and then $\kappa=2,(3,3)$, the wave function spreads out in $\left(x_{1}, x_{2}\right)$ space. Further increase of the barrier height causes the system to move into the insulator limit, plot $(4,3)$, forming two isolated lobes in the upper-right and lower-left quadrants. The eigenstate, in this case, corresponds to the physical situation of both particles residing in either the left well or the right well.

Finally, the third-excited state is illustrated in Fig. 10. In contrast to $\Psi_{1}$ and $\Psi_{2}$, this eigenstate is of even parity such 


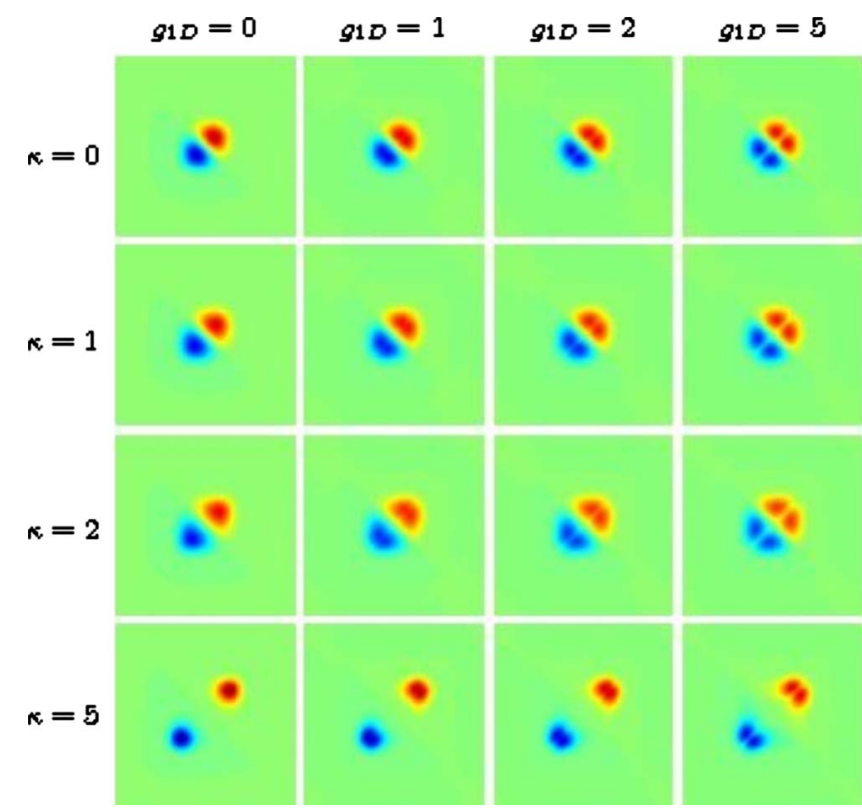

FIG. 9. (Color online) Second-excited eigenfunction, $\Psi_{2}\left(x_{1}, x_{2}\right)$, for a pair of particles in a double-well potential. The different columns represent different values for the interaction coupling and the effect of varying the barrier height is illustrated down a given column. The same values for the parameters $g_{1 \mathrm{D}}$ and $\kappa$, as considered in Fig. 8, are examined here. Each individual plot spans the range $-5.6<x_{1}, x_{2}<5.6$, where the quantities $x_{1}, x_{2}, \kappa$, and $g_{1 \mathrm{D}}$ are given in the scaled units introduced in Sec. II.

that $\Psi_{3}\left(x_{1}, x_{2}\right)=\Psi_{3}\left(-x_{1},-x_{2}\right)$. Scanning down column 1: As the system moves into the insulator limit, the eigenstate is composed of four equally weighted lobes in the four quadrants, equivalent to the corresponding ground-state eigenfunction, seen in Fig. $3(4,1)$. In fact from Fig. 2(a), in the noninteracting case, the four lowest eigenstates all become degenerate in the insulator limit $(\kappa \rightarrow \infty)$. As a consequence, the eigenfunction plot $(4,1)$ in Figs. 3 and $8-10$ relate to four degenerate states.

This symmetric eigenstate is nonzero along the line $x_{1}$ $=x_{2}$. As one increases $g_{1 \mathrm{D}}$ one again observes the exclusion of the wave function from this line (e.g., examining row 1 in Fig. 10). As barrier height is increased the wave function expands in $\left(x_{1}, x_{2}\right)$ space and there is some suppression of the wave function in the region of the rising barrier (i.e., $x_{1}=0$ and $\left.x_{2}=0\right)$. In the insulator limit, e.g., in plot $(4,4)$ for which $\kappa=5$, the wave function in the off-diagonal quadrants vanishes and one observes two double lobes in the lower-left and upper-right quadrants, representing the physical situation where both particles reside in the same well. The degeneracy of $\Psi_{2}$ and $\Psi_{3}$, in the limit $\kappa \rightarrow \infty$ (seen in Fig. 2) is manifested in the corresponding wave-function plots. This is demonstrated by comparing corresponding plots in the bottom rows $(\kappa=5)$ of Figs. 9 and 10 .

In the limit of large $\kappa$ (and for any positive interaction), the ground and first-excited states correspond to the two particles in separate wells. By contrast, the second- and thirdexcited states, in the same limit, correspond to two particles in the same well. It follows that an increase in the repulsive interaction coupling will cause this second pair of levels to

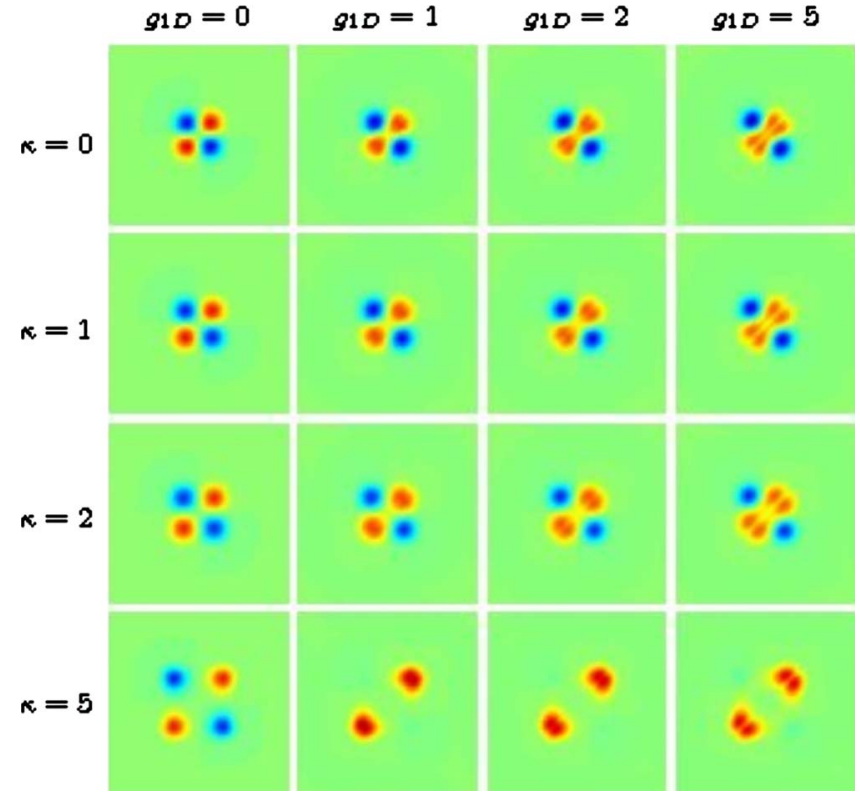

FIG. 10. (Color online) Third-excited eigenfunction, $\Psi_{3}\left(x_{1}, x_{2}\right)$, for a pair of particles in a double-well potential. The different columns represent different values for the interaction coupling and the effect of varying the barrier height is illustrated down a given column. The same values for the parameters $g_{1 \mathrm{D}}$ and $\kappa$, as considered in Fig. 8, are examined here. Each individual plot spans the range $-5.6<x_{1}, x_{2}<5.6$, where the quantities $x_{1}, x_{2}, \kappa$, and $g_{1 \mathrm{D}}$ are given in the scaled units introduced in Sec. II.

be shifted upwards in energy. In this way, in the $\kappa \rightarrow \infty$ limit, one observes the separation of these two pairs of levels to increase as $g_{1 \mathrm{D}}$ is increased (see Fig. 2). The increasing separation of these levels with increasing $\kappa$ is corollary to this. As $\kappa$ is increased the particles become more tightly confined to the individual wells. This increased confinement, for the upper pair of levels, will give rise to an increased interaction of the two particles and a subsequent increase in the energy of these eigenstates, relative to the lower pair.

\section{B. Momentum distribution}

The momentum distributions for the excited states are calculated as outlined in Sec. V A. The calculated distributions for the second-excited state $\left(\Psi_{2}^{o}\right.$, where the superscript "o" indicates the "odd" inversion symmetry of this eigenstate) are displayed in Fig. 11. For $\kappa=0$ one observes a doublehumped distribution that becomes narrower with increasing $\kappa$ and, in the insulator limit, gives way to a single-peak distribution with high-energy tails. This is similar to the result for $\Psi_{1}^{o}$ (not shown). An increase in the interaction coupling has the effect of narrowing the momentum distribution. Figure 9 illustrates that increasing $g_{1 \mathrm{D}}$ will expand the wave function in $\left(x_{1}, x_{2}\right)$ space, leading to this reciprocal narrowing in momentum space. At the same time the increased interaction leads to an accentuation of the double-peaked structure, observed for small $\kappa$.

Figure 12 illustrates the momentum distribution for the even-parity state $\Psi_{3}^{\mathrm{e}}$. For the noninteracting case, $g_{1 \mathrm{D}}=0$ (a), a double-mode distribution arises with a node at $k=0$. This 


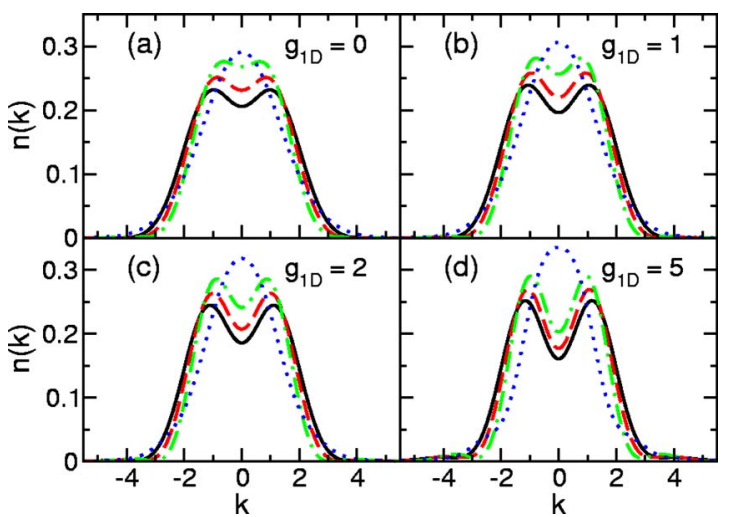

FIG. 11. (Color online) Momentum distribution for the secondexcited state $\left(\Psi_{2}^{0}\right)$ for a system of two atoms confined by the double-well potential $V(x)=\left(x^{4}-\kappa x^{2}\right)$. Four different values of interaction strength are considered: (a) $g_{1 \mathrm{D}}=0$, (b) $g_{1 \mathrm{D}}=1$, (c) $g_{1 \mathrm{D}}$ $=2$, and $g_{1 \mathrm{D}}=5$. In each figure the effect of varying the barrier height is also illustrated. Barrier heights considered are $\kappa=0$ (solid line), $\kappa=1$ (dashed line), $\kappa=2$ (dotted-dashed line), and $\kappa=5$ (dotted line). The momentum distribution is identical to that of $\Psi_{1}^{\mathrm{o}}$ in the noninteracting limit. Increasing the interaction strength $\left(g_{1 \mathrm{D}}\right)$ leads to an increasingly peaked distribution.

node is accounted for due to the separable nature of $\Psi_{3}^{\mathrm{e}}$ in the noninteracting limit: $\Psi_{3}^{\mathrm{ni}}\left(\kappa ; x_{1}, x_{2}\right)=u_{1}\left(\kappa ; x_{1}\right) u_{1}\left(\kappa ; x_{2}\right)$. Considerable narrowing of this distribution is noted as $\kappa$ is increased and in the insulator limit a second pair of smaller peaks emerges. This second pair of peaks may be viewed as interference fringes from each particle being distributed between the two wells-compare Fig. $3(4,1)$ and Fig. $10(4,1)$.

For increased interaction strength $\left(g_{1 \mathrm{D}}\right)$ one continues to observe the narrowing of the distribution with increased barrier height. However, the presence of the interactions causes

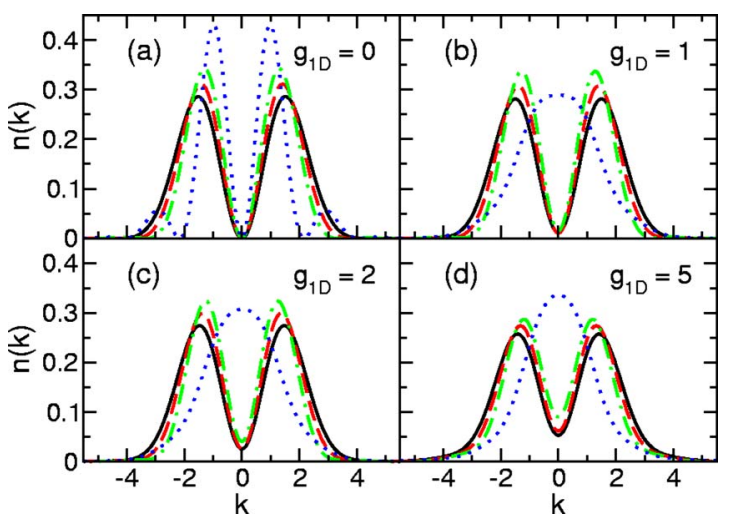

FIG. 12. (Color online) Momentum distribution for the thirdexcited state $\left(\Psi_{3}^{\mathrm{e}}\right)$ for a system of two atoms confined by the double-well potential $V(x)=\left(x^{4}-\kappa x^{2}\right)$. Four different values of interaction strength are considered: (a) $g_{1 \mathrm{D}}=0$, (b) $g_{1 \mathrm{D}}=1$, (c) $g_{1 \mathrm{D}}$ $=2$, and $g_{1 \mathrm{D}}=5$. In each figure the effect of varying the barrier height is also illustrated. Barrier heights considered are $\kappa=0$ (solid line), $\kappa=1$ (dashed line), $\kappa=2$ (dotted-dashed line), and $\kappa=5$ (dotted line). The separable nature of $\Psi_{3}^{\mathrm{e}}$ in the noninteracting limit, as seen in Eq. (14), leads to a node in momentum distribution for $k$ $=0$. Introduction of interactions means that $\Psi_{3}^{\mathrm{e}}$ is no longer separable and the node is no longer enforced.

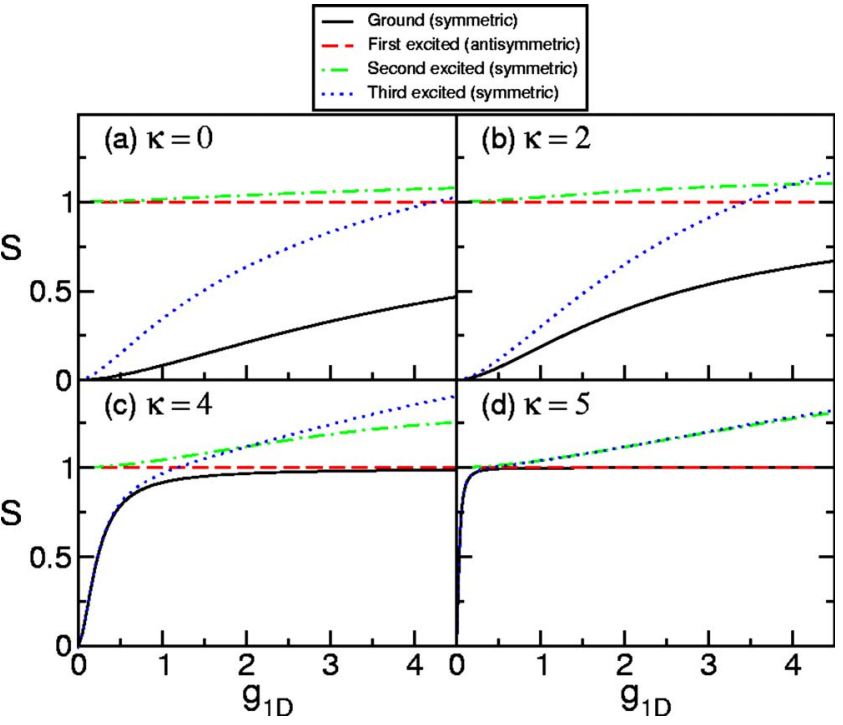

FIG. 13. (Color online) von Neumann entropy, $S$, as a function of the interaction strength $\left(g_{1 \mathrm{D}}\right)$ for the four lowest two-particle states: $\Psi_{0}^{\mathrm{e}}$ (solid line), $\Psi_{1}^{\mathrm{o}}$ (dashed line), $\Psi_{2}^{\mathrm{o}}$ (dotted-dashed line), and $\Psi_{3}^{\mathrm{e}}$ (dotted line). The dependence is also illustrated for a number of different barrier heights: $\kappa=0$ (a), $\kappa=2$ (b), $\kappa=4$ (c), and $\kappa$ $=5(\mathrm{~d})$.

the node at $k=0$ to be removed, as one can no longer write the eigenfunction in the separable form given in Eq. (14). Instead one just observes a strong depression of the distribution about $k=0$. At the same time, the introduction of the interactions has the effect of completely removing the double-peaked structure in the insulator limit, as is observed for the dotted line $(\kappa=5)$ in each of Figs. 12(b)-12(d). As seen in Fig. 10, in the presence of a finite interaction the wave function in the off-diagonal quadrants vanishes in the insulator limit, and this eigenstate describes a situation where both particles occupy one side of the double-well.

\section{C. von Neumann entropy}

As for the ground state, one may obtain the von Neumann entropy for the excited states of the two-particle system via diagonalization of the reduced single-particle density matrix. In this section the dependence of the von Neumann entropy, $S$, of the four lowest two-particle states, on the interaction strength $\left(g_{1 \mathrm{D}}\right)$ and the barrier height $(\kappa)$ is considered.

\section{Variation of von Neumann entropy with interaction strength}

Figure 13 illustrates the dependence of $S$ on the interaction coupling, $g_{1 \mathrm{D}}(>0)$. The dependence is examined for four different values of barrier height: $\kappa=0$ (a), $\kappa=2$ (b), $\kappa$ $=4(\mathrm{c})$, and $\kappa=5(\mathrm{~d})$. For each value of the barrier height the entropy of the four lowest eigenstates is depicted: Ground state (solid line), first-excited state (dashed line), secondexcited state (dotted-dashed line), and third-excited state (dotted line). The dependence of the ground-state entropy on $g_{1 \mathrm{D}}$ has already been examined in Fig. 5, however it is useful to replicate these plots here to help inform the examination of the excited-state plots. 
Several important features are noted. In all cases the firstexcited state (dashed line) shows no dependence on the interaction strength, as is expected owing to the symmetry of this eigenstate. Instead, this eigenstate exhibits a value of $S$ $=1$ for all $g_{1 \mathrm{D}}$. This value follows from the analytic form for this eigenstate, $\Psi_{1}^{\mathrm{ni}}$, given by Eq. (14), which holds for all values of $g_{1 \mathrm{D}}$. At the same time, the analytic representations for the three remaining eigenstates are also given in Eq. (14), for $g_{1 \mathrm{D}}=0$. From these representations it is clear that, in the noninteracting limit, the entropy for the ground state (solid line) and third-excited state (dotted line) is always zero, as these states may always be represented as direct-product states for $g_{1 \mathrm{D}}=0$. In a similar way, the second-excited state (dotted-dashed line) always assumes a value of $S=1$ in the noninteracting limit. Once again, this may be attributed to the symmetrized form for this state as given by $\Psi_{2}^{\mathrm{ni}}$ in Eq. (14).

Considering the case of $\kappa=0$, Fig. 13(a), the ground state begins at $S=0$ and increases monotonically with $g_{1 \mathrm{D}}$. As $g_{1 \mathrm{D}} \rightarrow \infty$ one enters the TG regime and this ground state (solid line) becomes degenerate with the first-excited (dashed line) state and $S \approx 1$. By contrast, the second-excited state begins with $S=1$, as discussed, and increases with increasing $g_{1 \mathrm{D}}$, but at a much slower rate than that exhibited by the ground state. The third-excited state (dotted line) begins, like the ground state, with $S=0$ and increases rapidly with increasing interaction strength.

Increasing the height of the barrier to $\kappa=2$, Fig. 13(b), one observes qualitatively similar behavior from all four states except that each state exhibits a more marked variation in $S$ over the range of $g_{1 \mathrm{D}}$ examined. As one moves into the insulator regime, e.g., $\kappa=4$, Fig. 13(c), the behavior changes quite significantly. As discussed previously, the ground state exhibits a very drastic variation with $g_{1 \mathrm{D}}$, converging very rapidly to $S \approx 1$. The second-excited state still exhibits the same basic behavior as noted for smaller $\kappa$ but, once again, the increased barrier height leads to an increased sensitivity of this state to variation in $g_{1 \mathrm{D}}$. The third-excited state shows a distinct change in behavior for this increased barrier height. At small values of interaction coupling $\left(g_{1 \mathrm{D}}<1\right)$ the entropy of this state follows closely that of the ground state. As interaction strength is increased beyond this value then the ground-state entropy begins to plateau at $S \approx 1$, while that of third-excited state continues to increase. Increasing the barrier height to $\kappa=5$, Fig. 13(d), moves the system deeper into the insulator limit and the behavior demonstrated in (c) becomes even more striking. In this case the behavior of the ground-state entropy is more dramatic, with the entropy saturating at $S \approx 1$, already, for $g_{1 \mathrm{D}} \approx 0.5$. Again the entropy of the third-excited state follows this trend identically. However, where the entropy of the ground-state plateaus at $S$ $\approx 1$, the entropy of the third-excited state continues to increase and follows now, almost identically, the entropy of the second-excited state. A handle on this behavior is provided by the wave-function plots of Figs. 3, 9, and 10. One observes that, in this insulator limit $(\kappa=5)$, the third-excited state, for small $g_{1 \mathrm{D}}$, as seen in Fig. $10(4,1)$, closely resembles the ground state in Fig. $3(4,1)$. For larger interaction couplings $\left(g_{1 \mathrm{D}} \geq 1\right)$ the eigenfunction for this thirdexcited state, as seen in Fig. $10(4,2)-(4,4)$, closely

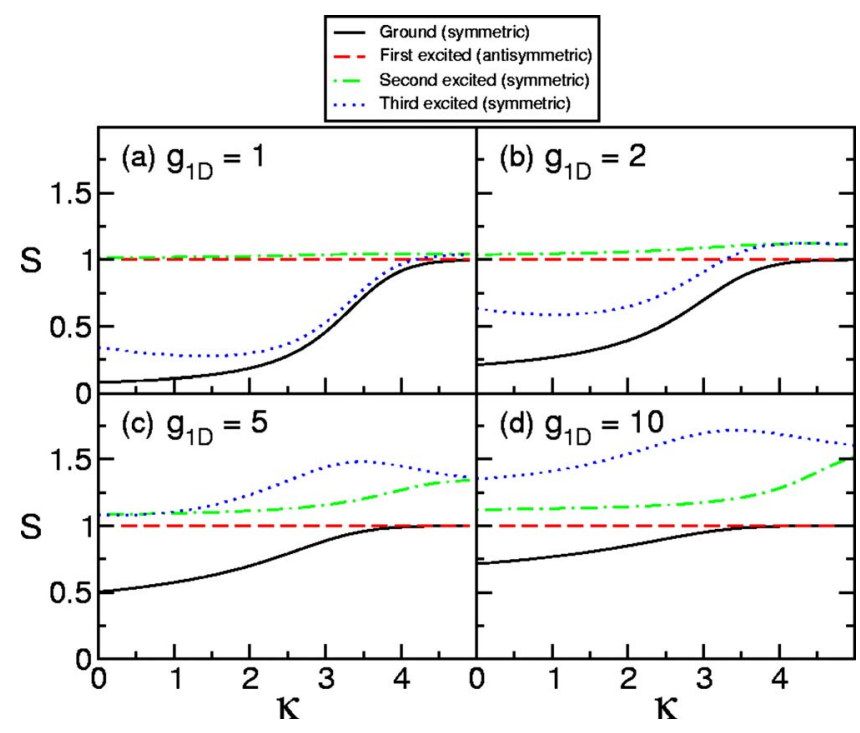

FIG. 14. (Color online) von Neumann entropy, $S$, as a function of the barrier height $(\kappa)$ for the four lowest two-particle states: $\Psi_{0}^{\mathrm{e}}$ (solid line), $\Psi_{1}^{\mathrm{o}}$ (dashed line), $\Psi_{2}^{\mathrm{o}}$ (dotted-dashed line), and $\Psi_{3}^{\mathrm{e}}$ (dotted line). The dependence is examined for a number of different interaction coupling strengths: (a) $g_{1 \mathrm{D}}=1$, (b) $g_{1 \mathrm{D}}=2$, (c) $g_{1 \mathrm{D}}=5$, and (d) $g_{1 \mathrm{D}}=10$.

resembles that of the second-excited state in Fig. $9(4,2)$ $(4,4)$.

\section{Variation of von Neumann entropy with barrier height}

The variation of von Neumann entropy with barrier height is illustrated in Fig. 14, for the same four, lowest-energy two-particle states. In this case, four different values of interaction coupling are presented: $g_{1 \mathrm{D}}=1(\mathrm{a}), g_{1 \mathrm{D}}=2(\mathrm{~b})$, $g_{1 \mathrm{D}}=5(\mathrm{c})$, and $g_{1 \mathrm{D}}=10(\mathrm{~d})$. Again, in each plot the eigenstates are represented by the same line types used in Fig. 13.

Some general features and behaviors can be noted from these plots. Again, the first-excited state is observed to have an entropy of unity for all $g_{1 \mathrm{D}}$ and $\kappa$. For $\kappa \rightarrow \infty$, the entropy of the ground state tends to a value of unity, regardless of the value of $g_{1 \mathrm{D}}$ (provided $g_{1 \mathrm{D}}>0$ ). In this limit the ground state of the system is described by one particle in each one-half of the double-well potential, and corresponds to the Mottinsulator regime. On the other hand, the initial value of $S$ (when $\kappa=0$ ) is sensitive to $g_{1 \mathrm{D}}$. The higher the value of $g_{1 \mathrm{D}}$, the larger is the initial value of $S$. As $S \rightarrow 1$ in the insulator limit, it follows that the entropy of the ground state exhibits a less dramatic variation with $\kappa$, for larger values of interaction strength. For all of the symmetric eigenstates, i.e., ground (solid line), second excited (dotted-dashed line), and third excited (dotted line), as the interaction strength is increased the entropy of the eigenstates, in general, increases, consistent with Fig. 13. In particular, the entropy of these symmetric states in the absence of a barrier $(\kappa=0)$, increases with increasing $g_{1 \mathrm{D}}$. The second-excited state (dotted-dashed line) exhibits an entropy that monotonically increases with $\kappa$ for the range of parameter space considered. By contrast, the third-excited state (dotted line) exhibits an entropy that both increases then decreases with raising of the barrier. 
One will also note that in the limit of large barrier heights (i.e., $\kappa \rightarrow \infty$ ), the entropy of the second- and third-excited states tend to the same value. Although not obvious from Fig. 14(d), this fact has also been verified for the case of $g_{1 \mathrm{D}}=10$. Once again, a handle on why this happens can be obtained from the wave function plots for these eigenstates in Figs. 9 and 10. One can see that in the presence of finite interactions, these two eigenstates become identical in the insulator limit, except for some phase (compare row 4 of these figures). Figure 14 also suggests that the value of $S$ to which these two states converge, in the insulator limit, is greater than 1 and increases with increasing interaction strength, $g_{1 \mathrm{D}}$.

This behavior of the entropy may be qualitatively understood as follows. Both the second- and third-excited states correspond, in the insulator limit, to the physical situation of two particles coexisting in either the right-hand well or the left-hand well. As such, these states may be roughly represented by Bell-type states of the form $1 / \sqrt{2}(|20\rangle \pm|02\rangle)$ - see Sec. V B 3 for the definition of these basis states. Such a Bell state carries one e-bit of entanglement, with a corresponding von Neumann entropy of unity. However, beyond this, there are also correlations between the two particles coexisting in the same well, as can be seen, for example, from Fig. $10(4,4)$. Here the repulsive interactions between the particles occupying the same well leads to a partition of the wave function, within each well, into two lobes. Considering the double lobe seen in the upper-right quadrant, corresponding to both particles coexisting in the right-hand well of the double-well. The upper onehalf of the lobe represents the situation where particle 1 is on the left-hand side of this well and particle 2 is on the righthand side, the lower one-half lobe corresponds to the reverse of this situation $\left(x_{1} \leftrightarrow x_{2}\right)$. In this case the correlations in the system are analogous to the correlations that are observed for the ground state, $\Psi_{0}$, in the absence of any barrier $(\kappa=0)$. These correlations (and therefore $S$ ) are seen to increase as the interaction coupling is increased. One significant distinction exists between these single-well correlations, seen in states $\Psi_{2,3}$, and the correlations seen in the ground state, for $\kappa=0$. On increasing $\kappa$, the second- and third-excited states tend to become more confined and the two-particle wave function becomes increasingly localized in the single well. However, the particle-particle interactions will compete with this effect, attempting to keep the two-particle wave function spread in space and, in particular, minimized along the line $x_{1}=x_{2}$. For the ground state this particular type of single-well competition between $\kappa$ and $g_{1 \mathrm{D}}$ is not experienced. So, in the insulator limit, the second- and third-excited states will have correlations arising from the realization of the Bell-type state, and the single-well correlations due to the two interacting particles coexisting in the same well. This combination of factors leads to an entropy which is greater than unity, with the contribution of the single-well correlations, in general, increasing with increasing interaction strength.

\section{Stimulating two-particle excitations}

The previous results have clearly illustrated that manipulations of this two-particle system can be achieved through

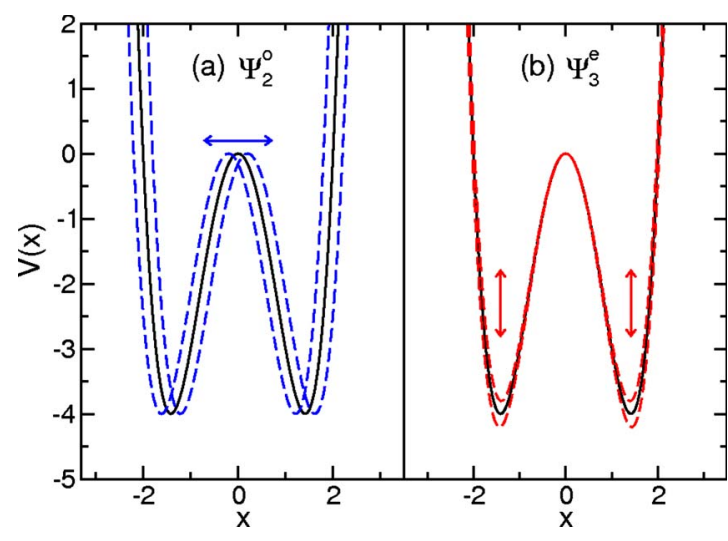

(c)

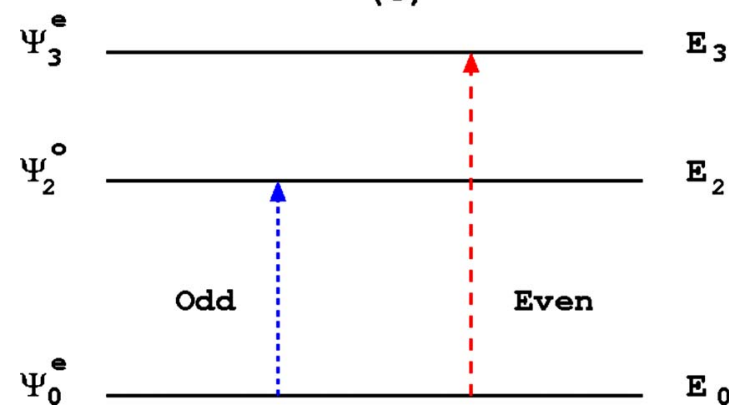

FIG. 15. (Color online) Time-dependent manipulation of the two-particle state may be achieved by (a) oscillation of the trap from side to side or (b) the modulation of the barrier height. The former process provides a dipole coupling between the ground state, $\left|\Psi_{0}^{\mathrm{e}}\right\rangle$, and the state $\left|\Psi_{2}^{\mathrm{o}}\right\rangle$. The second scheme will provide a quadrupole coupling between the ground state and the state $\left|\Psi_{3}^{\mathrm{e}}\right\rangle$. (c) A schematic energy level representation for these processes.

the variation of the control parameters $g_{1 \mathrm{D}}$ and $\kappa$ in some adiabatic manner. However, one could also consider timedependent manipulation of the state. Considering the insulator limit, one may propose two methods of coupling these lowest levels: (a) Shaking the trap from side-to-side and (b) modulating the barrier height (see Fig. 15). To first-order, the former represents a dipole excitation, capable of coupling $\left|\Psi_{0}^{\mathrm{e}}\right\rangle$ and $\left|\Psi_{2}^{\mathrm{o}}\right\rangle$. The latter scheme (to first order) corresponds to a quadrupole excitation, capable of coupling states $\left|\Psi_{0}^{\mathrm{e}}\right\rangle$ and $\left|\Psi_{3}^{\mathrm{e}}\right\rangle$. In this way, by employing such techniques it should prove possible to exploit these three lowest eigenstates in order to engineer the two-particle state in a timedependent fashion.

Further investigation of this idea of time-dependent manipulation of the two-particle state could prove a useful extension to the present study. In particular, a combination of time-dependent excitation processes and the adiabatic variation of control parameters, $g_{1 \mathrm{D}}$ and $\kappa$, should permit an impressive degree of control over the two-particle state, within this system.

\section{SUMMARY}

The system of two interacting particles in a prototypical double-well potential of the form $V(x)=A\left(x^{4}-\kappa x^{2}\right)$ has been 
considered. Using a Cartesian DVR, the eigenspectrum for this system has been studied and the four lowest eigenstates have been obtained and investigated for varying barrier height and interaction strength. For each state the twoparticle eigenfunction, the momentum distribution, and the von Neumann entropy have been examined. It was found that the ground state for this double-well system exhibits behavior that closely resembles that observed in a previous study of the $\delta$-split trap potential [34]. In particular, the ground-state wave function is suppressed along the lines $x_{1}$ $=0$ and $x_{2}=0$ as barrier height is increased, leading to a quadrant separation of the wave function. In the presence of repulsive interactions $\left(g_{1 \mathrm{D}}>0\right)$ only the contributions in the off-diagonal quadrants remain in the insulator limit $(\kappa \rightarrow \infty)$. In this limit the ground state of the system is composed of one particle in each one-half of the double-well. The momentum distributions display an initial narrowing with increasing barrier height but with a broadening and high-energy wings being observed in the insulator limit. Furthermore, the secondary peaks observed in the momentum distribution for the double-well, in the noninteracting regime, are quickly suppressed in the presence of repulsive interactions. The variation in the von Neumann entropy $(S)$ with interaction strength shows remarkably similar behavior. In all cases $S$ $=0$ in the absence of interactions and for $g_{1 \mathrm{D}} \rightarrow \infty, S$ saturates at a value close to unity. Increasing the height of the barrier, in each case, has the effect of making the entropy more sensitive to changes in the interaction strength, around $g_{1 \mathrm{D}}=0$. Similarly, the behavior of the entropy with varying barrier height exhibits generic features between the two double-well systems. In both cases the ground-state entropy saturates at a value of unity as $\kappa \rightarrow \infty$, regardless of the value of $g_{1 \mathrm{D}}$. The initial value of $S$ (i.e., the value of $S$ for $\kappa=0$ ) is determined by the strength of the interaction, with larger interaction coupling leading to larger initial entropy. As such, the sensitivity of $S$ to $\kappa$ is reduced for double-well systems with larger interaction couplings $\left(g_{1 \mathrm{D}}\right)$. This behavior of the ground-state entropy is also illustrated within a Bose-Hubbard model, wherein the controllable parameters are the on-site interaction $(U)$ and the tunneling strength $(J)$.

As well as examining the ground state of this double-well system, some of the properties of the three lowest excited states have also been studied. Two of these states are found to be symmetric while one is antisymmetric and they constitute the lowest band of the two-particle, double-well system. The antisymmetric state is found to be completely independent of the interaction parameter $\left(g_{1 \mathrm{D}}\right)$. However, this state displays a dependence on the barrier height and in the limit of a high barrier becomes degenerate with the ground statecorresponding, physically, to the situation of each particle residing in a separate, isolated well. The von Neumann entropy for this antisymmetric state is identically equal to one for all $\kappa$ and $g_{1 \mathrm{D}}$.

The second- and third-excited states are symmetric. In the insulator limit (provided $g_{1 \mathrm{D}}>0$ ) the states become degenerate and correspond to the physical situation where both particles occupy the same well. Both eigenstates demonstrate momentum distributions that are double-humped, with the double-hump giving way to a single peak in the insulator limit. For $g_{1 \mathrm{D}}=0$, the third-excited state exhibits secondary peaks in the momentum distribution, similar to the ground state in the noninteracting regime. The entropy of both states increases with $g_{1 \mathrm{D}}$, with that of the third-excited state showing a more marked variation. As for the ground state, increasing barrier height $\kappa$ has the effect of increasing the sensitivity of the entropy to variations in $g_{1 \mathrm{D}}\left(\right.$ about $\left.g_{1 \mathrm{D}}=0\right)$. In the insulator limit the entropy of the third-excited state is found to follow, almost identically, that of the ground state for small $g_{1 \mathrm{D}}$. As the ground-state entropy saturates at $S$ $\approx 1$, the entropy of the third-excited state continues to increase and, for larger $g_{1 \mathrm{D}}$, follows, almost identically, that of the second-excited state. Indeed, in the insulator limit and for fixed interaction strength, the second- and third-excited states are found to have the same entropy (as follows from the physical equivalence of these states in this limit). The entropy, in this case, is proposed to have two contributions due to (i) the realization of a Bell-type state with both particles co-occupying either the left-hand or right-hand well and (ii) single-well correlations, owing to the repulsive interaction of the two particles occupying the same well.

Outlook. The double-well arrangement studied in this work represents a more experimentally realizable system, compared to the $\delta$-split trap previously considered. Having characterized the properties of the ground and lowest-excited eigenstates, the foundation is laid for future investigation into state manipulations using this system. Future avenues may include the time-dependent manipulation of states through shaking of the trap, an oscillating barrier height or introduction of a constant, or oscillating, field gradient. These time-dependent manipulations, along with the adiabatic variation of the control parameters $g_{1 \mathrm{D}}$ and $\kappa$, should allow for comprehensive state engineering within the lowest band of this two-particle system.

\section{ACKNOWLEDGMENTS}

The authors would like to thank John Goold, Thomas Busch, and Mauro Paternostro for helpful discussions. D.S.M. would like to acknowledge funding from the Department for Employment and Learning (NI) and the support of the Sorella Trust (NI).
[1] C. Cohen-Tannoudji, Rev. Mod. Phys. 70, 707 (1998).

[2] M. Anderson et al., Science 269, 198 (1995).

[3] K. B. Davis, M. O. Mewes, M. R. Andrews, N. J. van Druten, D. S. Durfee, D. M. Kurn, and W. Ketterle, Phys. Rev. Lett.
75, 3969 (1995).

[4] F. Dalfovo, S. Giorgini, L. Pitaevskii, and S. Stringari, Rev. Mod. Phys. 71, 463 (1999).

[5] B. Anderson and M. Kasevich, Science 282, 1686 (1998). 
[6] F. Cataliotti et al., Science 293, 843 (2001).

[7] I. Bloch, Nat. Phys. 1, 23 (2005).

[8] I. Bloch, J. Phys. B 38, S629 (2005).

[9] C. Monroe, Nature (London) 416, 238 (2002).

[10] D. Jaksch, H.-J. Briegel, J. I. Cirac, C. W. Gardiner, and P. Zoller, Phys. Rev. Lett. 82, 1975 (1999).

[11] O. Mandel et al., Nature (London) 425, 937 (2003).

[12] M. P. A. Fisher, P. B. Weichman, G. Grinstein, and D. S. Fisher, Phys. Rev. B 40, 546 (1989).

[13] D. Jaksch, C. Bruder, J. I. Cirac, C. W. Gardiner, and P. Zoller, Phys. Rev. Lett. 81, 3108 (1998).

[14] M. Greiner, O. Mandel, T. Esslinger, T. Hänsch, and I. Bloch, Nature (London) 415, 39 (2002).

[15] K. Huang, Statistical Mechanics, 2nd ed. (Wiley, New York, 1987).

[16] T. Busch, Found. Phys. 28, 549 (1998).

[17] Z. Idziaszek and T. Calarco, Phys. Rev. A 71, 050701(R) (2005).

[18] M. Block and M. Holthaus, Phys. Rev. A 65, 052102 (2002).

[19] E. Tiesinga, C. J. Williams, F. H. Mies, and P. S. Julienne, Phys. Rev. A 61, 063416 (2000).

[20] E. L. Bolda, E. Tiesinga, and P. S. Julienne, Phys. Rev. A 66, 013403 (2002).

[21] E. L. Bolda, E. Tiesinga, and P. S. Julienne, Phys. Rev. A 68, 032702 (2003).

[22] T. Kinoshita, T. Wenger, and D. Weiss, Science 305, 1125 (2004).

[23] B. Paredes et al., Nature (London) 429, 277 (2004).

[24] W. Ketterle and N. van Druten, Phys. Rev. A 54, 656 (1996).

[25] A. Görlitz et al., Phys. Rev. Lett. 87, 130402 (2001).

[26] M. Olshanii, Phys. Rev. Lett. 81, 938 (1998).

[27] L. Tonks, Phys. Rev. 50, 955 (1936).

[28] M. Girardeau, J. Math. Phys. 1, 516 (1960).
[29] V. Yukalov and M. Girardeau, Laser Phys. Lett. 2, 375 (2005).

[30] M. D. Girardeau and E. M. Wright, Phys. Rev. Lett. 84, 5691 (2000).

[31] M. D. Girardeau, E. M. Wright, and J. M. Triscari, Phys. Rev. A 63, 033601 (2001).

[32] T. Busch and G. Huyet, J. Phys. B 36, 2553 (2003).

[33] Y. Lin and B. Wu, Phys. Rev. A 75, 023613 (2007).

[34] D. S. Murphy, J. F. McCann, J. Goold, and T. Busch, Phys. Rev. A 76, 053616 (2007).

[35] D. Baye and P.-H. Heenen, J. Phys. A 19, 2041 (1986).

[36] J. Light and J. T. Carrington, Adv. Chem. Phys. 114, 263 (2000).

[37] S. Zöllner, H.-D. Meyer, and P. Schmelcher, Phys. Rev. A 74, 053612 (2006)

[38] S. Zöllner, H.-D. Meyer, and P. Schmelcher, Phys. Rev. A 74, 063611 (2006).

[39] S. Zöllner, H.-D. Meyer, and P. Schmelcher, Phys. Rev. A 75, 043608 (2007).

[40] K. Burnett, P. Julienne, P. Lett, E. Tiesinga, and C. Williams, Nature (London) 416, 225 (2002).

[41] A. Coleman and V. Yukalov, Reduced Density Matrices (Springer, New York, 2000).

[42] R. Paškauskas and L. You, Phys. Rev. A 64, 042310 (2001).

[43] Y. S. Li, B. Zeng, X. S. Liu, and G. L. Long, Phys. Rev. A 64, 054302 (2001).

[44] B. Sun, D. L. Zhou, and L. You, Phys. Rev. A 73, 012336 (2006).

[45] G. Ghirardi and L. Marinatto, Fortschr. Phys. 51, 379 (2003).

[46] G. C. Ghirardi and L. Marinatto, Phys. Rev. A 70, 012109 (2004).

[47] G. Ghirardi and L. Marinatto, Fortschr. Phys. 52, 1045 (2004).

[48] D. Cavalcanti, L. M. Malard, F. M. Matinaga, M. O. Terra Cunha, and M. F. Santos, Phys. Rev. B 76, 113304 (2007). 